\title{
A friendly location-aware system to facilitate the work of technical directors when broadcasting sport events
}

\author{
Sergio Ilarri ${ }^{\mathrm{a}, *}$, Eduardo Mena ${ }^{\mathrm{a}}$, Arantza Illarramendi ${ }^{\mathrm{b}}$, Roberto Yus ${ }^{\mathrm{a}}$, Maider Laka ${ }^{\mathrm{c}}$ and \\ Gorka Marcos ${ }^{\mathrm{c}}$ \\ ${ }^{a}$ Department of Computer Science and Systems Engineering, University of Zaragoza, Zaragoza, Spain \\ ${ }^{\mathrm{b}}$ Basque Country University, San Sebastián, Spain \\ ${ }^{\mathrm{c}}$ Vicomtech Research Center, San Sebastián, Spain
}

\begin{abstract}
The production costs of broadcasting sport events that require tracking moving objects are continuously increasing. Although those events are very demanded by the audience, broadcasting organizations have economical difficulties to afford them. For that reason, they are demanding the development of new professional (software and hardware) equipments that lead to a considerable reduction of the production costs.

In this paper, we present a software system that takes into account these needs. This system allows a technical director to indicate his/her interest about certain moving objects or geographic areas in run-time. The system is in charge of selecting the cameras that can provide the types of views requested on those interesting objects and areas. So, it decreases the human effort needed to produce (create, edit and distribute) audiovisual contents, giving at the same time the opportunity to increase their quality. For this, the system provides a friendly interface to specify requirements and obtain which monitoring video cameras attached to moving or static objects fulfill them, along with a query processor to handle those requests in a continuous and efficient way. We illustrate the feasibility of our system in a specific scenario using real data of a traditional rowing race in the Basque Country.
\end{abstract}

Keywords: Mobile multi-camera management, location-aware systems, location-dependent queries, video broadcasting, sport events

\section{Introduction}

In the last years, different factors are provoking a deep revolution in the broadcast industry. First of all, there has been a strong decrease of the advertisement rates, mainly due to the economic crisis and the audience fragmentation caused by the digitalization and optimization of the spectrum (channel multiplication in satellite and terrestrial television) and the appearance of new communication platforms (e.g., video-blogs, Internet video platforms, mobile broadcasting, and so on). Secondly, continuous needs related to the upgrade of the technology (e.g., digitalization, development of media asset management systems, HD, 3D, etc.) are implying huge economic efforts. Finally, it is also evident that the expectations

${ }^{*}$ Corresponding author: Sergio Ilarri, University of Zaragoza, Department of Computer Science and Systems Engineering, Edificio Ada Byron, María de Luna 1, E-50018 Zaragoza, Spain. Tel.: +34 97676 23 40; Fax: +34 9767619 14; E-mail: silarri@unizar.es. 
of the audience are increasing. The quality and richness of the content (e.g., amazing views, last generation graphics, and so on) demanded by the audience and the advertisers is much bigger than in other distribution platforms such as Internet video platforms.

In such a context, organizations focus their efforts on the enrichment and the diversification of their offers but trying to reduce their production costs. On the one hand, the enrichment is tackled by the generation of very attractive and high-quality material, including in many cases technological support (e.g., the Obama hologram in the US presidential election in 2008). On the other hand, the reduction is considered by the acquisition of new professional products that, apart from begin able to deal with the last technology (e.g., IP interoperability, HD resolution, remote control mechanisms), either have a lower price due to the inclusion of hardware coming from the consumer electronic field or add new features that decrease the human effort required for content production. An example of this is the combination of professional cameras with low-cost cameras controlled remotely. This leads to an enrichment of the content consuming experience without having a big impact on the total number of cameramen required. However, that solution has a serious impact in one of the most complex and critical tasks in a live content production environment: the more cameras are employed, the more images are available, and therefore more complicated is for technical directors (people responsible for the content production of an event) to select the best video stream to broadcast.

The audiovisual production of rowing races in the Basque Country is a relevant paradigm of this situation and it will serve us as sample scenario in this paper. The live broadcasting of such rowing races requires a very complex infrastructure: one helicopter, sailing boats with cameras and GPS transmitters, more cameras in the harbor, and a production mobile unit (usually a trailer). In such a complex context, the technical director is responsible for the selection and coordination on the fly of the graphical material, video signals, and views that are finally broadcasted to the TV audience. These tasks become especially difficult when different unexpected events happen at different geographic areas or when different moving objects become interesting at the same time.

In order to help technical directors to obtain the best broadcasting results, we present in this paper a system that helps them to select the best candidate video signals coming from static or mobile cameras (i.e., cameras installed on rowing boats, other sailing boats, fixed locations, etc.). Our proposal, based on the LOQOMOTION system [19] extended with videocamera management, relies on mobile agent technology to bring the processing to the best place in the distributed wireless scenario, at any time. Thus, the camera selections provided by the system are updated continuously in an efficient manner, and it is possible to deal with different geographic areas of interest at the same time. The system can even alert about upcoming situations defined previously by the technical director (e.g., a certain object is within an area), or when some event happens (e.g., a camera gets close to a certain location). A preliminary version of our proposal appeared in [23], which has been improved and extended in this paper; among other extensions, we consider cameras that can both pan and tilt, and we analyze the precision of our system by testing a complete prototype using real GPS location data captured during a rowing race celebrated in September 2010.

The main contributions of our proposal are:

- We extend a general architecture for location-dependent query processing with videocamera management to help technical directors that broadcast sport events to deal with the multimedia information coming from different (static or moving) videocameras.

- The system enables technical directors to indicate his/her interest about certain (static or moving) objects or (fixed or moving) geographic areas predefined or defined in run-time. The system is in charge of selecting the cameras that can provide the types of views requested by the technical director on those interesting objects and areas. 


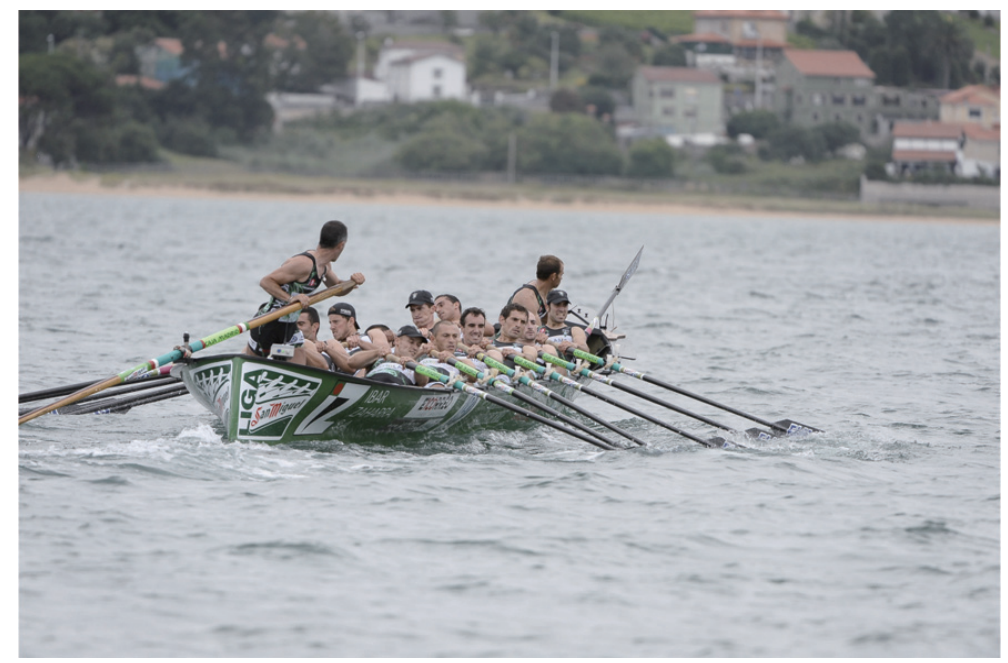

Fig. 1. "Kaiku" boat in a rowing race (image provided by courtesy of the Kaiku club).

- A flexible approach is followed, which can be applied to different distributed scenarios and requirements, and therefore new functionalities can be added to the system without compromising the main architecture.

The rest of the paper is structured as follows. In Section 2 we detail the features of the sample sport event used as motivating context and some interesting multimedia location-dependent queries that we would like to process automatically. In Section 3 we summarize the basic features of cameras and the work of a technical director. In Section 4 we introduce the concept of location-dependent queries, a general architecture for location-based query processing, and our proposal to extend it with the modeling and management of videocameras. In Section 5 we explain how queries in the sample scenario are processed by the proposed architecture. In Section 6 we present our prototype and some experiments that we have performed to validate our proposal. In Section 7 we review some related works. Finally, conclusions and future work are included in Section 8.

\section{Motivating context: Rowing races in San Sebastian}

Rowing racing (see Fig. 1) is a very popular sport in every seaside town of fishing tradition along the north of Spain. In the Basque Country this sport is very important, and almost every town has its own team, which always counts with the unconditional local support.

Although there are multiple competitions, the most important one is celebrated in the San Sebastian bay, once a year, since 1879. The boats leave from the harbor where they come back after making a turn in the sea, outside the protection of the bay, covering a total distance of 3 nautical miles. There are four lanes, although those lanes are only physically distinguished in the starting and turning points (see Fig. 2).

We have chosen this race due to the fact that it is a paradigmatic example of a potential live broadcasting event that can benefit from our work. In this race, celebrated during two consecutive weekends every September, the city of San Sebastian is crowded with many visitors, and the audience of the Basque TV broadcaster is very high. The technology and equipment involved in the event have been evolving during 


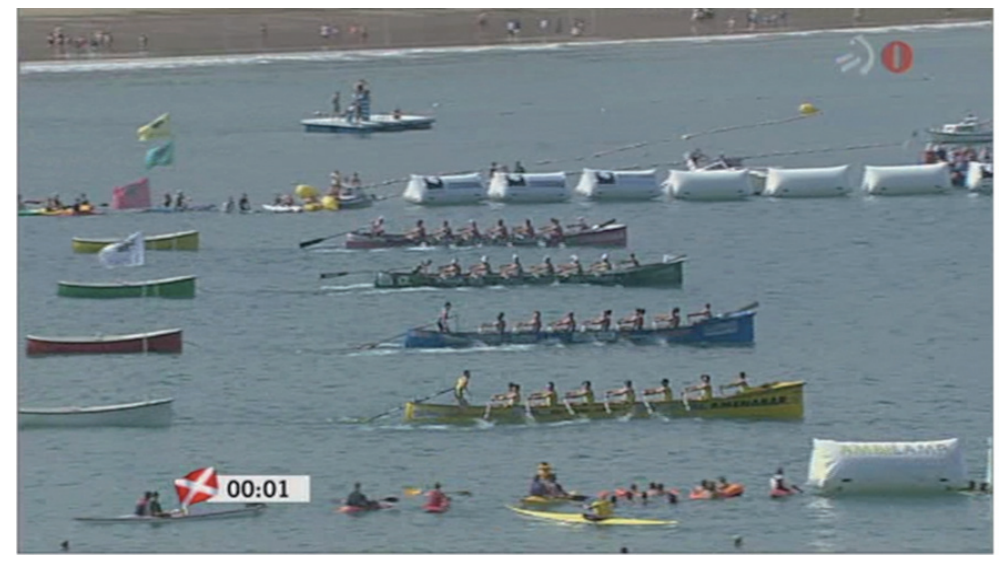

Fig. 2. Start of a rowing race (image provided by courtesy of the EiTB Media Group).

the last years and nowadays there are multiple cameras (on sailing boats, on a helicopter, in the harbor, on an island nearby, etc.), a GPS transmitter on every boat, a software application for the panel of judges to help them to determine the distance between the boats and the location of a boat with respect to its lane, a software tool to show on TV the real positions of the boats in a 3D reconstruction of the bay, microphones to capture the atmosphere sounds in different places, and so on.

In such a scenario, it is possible to attach a remotely controlled camera to each rowing boat. We would like to highlight that in many cases these cameras will provide innovative and interesting views from the content production perspective. For instance, these cameras may capture the exciting moment of the turning in the sea, the view of the audience perceived by the rowers, overtaking maneuvers, unusual ocean-side views of an island that is located in the middle of the bay, etc. Those views or points of interest can be classified into predefined ones (e.g., the view of spectators in the harbor) or defined while broadcasting (e.g., the view of an overtaking maneuver between two boats captured by the camera of a third boat). Figure 3 shows graphically some of the predefined points of interest on top of a 3D reconstruction of the race scenario.

However, it would be crucial to help the technical director to select among the available cameras those that can provide nice views, considering that the cameras could be remotely controlled and be continuously moving. It is important to emphasize that receiving the video signals is not a problem in this scenario, but the selection among the multiple video signals is a challenge. The system described in this paper helps the technical director in the identification and management of the best candidate videocameras that can view a specific (static or moving) area or object of interest; such target objects and areas can be predefined or specified during the broadcasting.

In the following, we enumerate some motivating queries that we would like to be answered and updated continuously:

1. Query 1: View a certain boat. The technical director could focus on a particular boat due to many reasons (it is the local team, it is leading the race, etc.), some of them caused by unexpected situations (an accident, a broken oar, etc.) which obviously should be captured quickly from any camera.

2. Query 2: Capture a close side view of any boat. The technical director could want to broadcast a close side view of some boat to show the big effort performed by its team of rowers during the race.

3. Query 3: Capture a wide view of the island from the ocean side. This is interesting for technical directors because this view of the island is usually very spectacular (it is a sheer cliff full of 


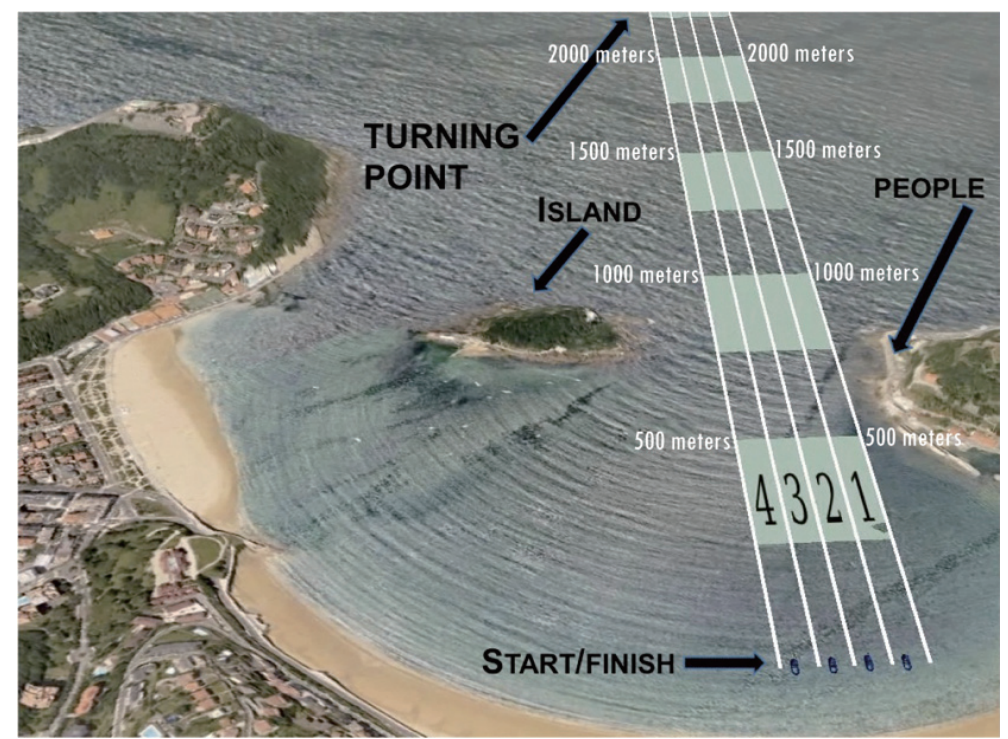

Fig. 3. Predefined points of in a rowing competition interest.

seagulls) and not as typical and easy-to-access as the well-known view of the island from the bay. For this query, technical directors are interested in cameras located far from the island (to capture it completely) but within a certain range (to get also a detailed picture). So, for this query, the locations of the cameras play an important role.

Other areas that are very interesting for technical directors, and therefore could be the target of similar queries, are: the ciaboga area (the turning point, which is a key part of the competition), the area of the harbor or the promenade at the seaside (usually crowded with people watching the race), etc. It would be beneficial to technical directors to be able to predefine areas that are usually relevant at some time during the race. However, the system should also allow technical directors to select, while broadcasting, any area of interest by dragging the mouse on a map.

\section{Technological context: Cameras and technical direction}

In this section, we describe the features of cameras and some basic ideas about the work of a technical director.

\subsection{The features of cameras}

Today, most of the low-cost/mid-range cameras employed in scenarios similar to the one described (e.g., F1 car races, sailing, etc.) offer a fixed view (e.g., a front view from the driver's perspective). From the production point of view, this implies important difficulties for the generation of attractive and rich content, mainly due to the limitation or lack of control of the rotation (pan and tilt) and zoom of the camera.

However, the electronics consumer sector is providing new cameras with very competitive prices that offer an acceptable image quality while providing rich remote control functionalities. Figure 4 shows some examples of these cameras, that allow a remote control of their parameters. 


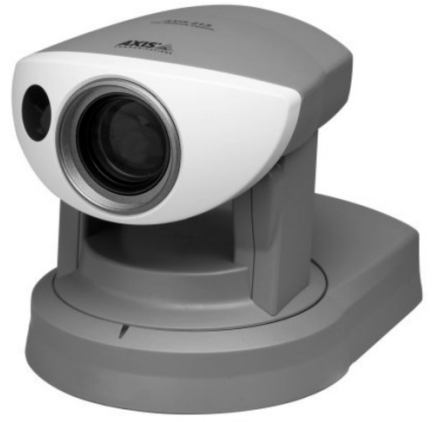

(a) Axis $213 \mathrm{PTZ}$

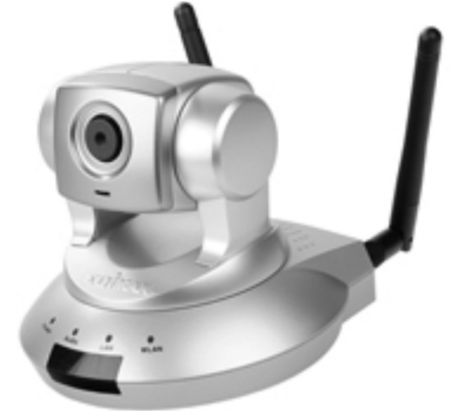

(b) Edimax IC-7000 PTn

Fig. 4. Remote motorized cameras.

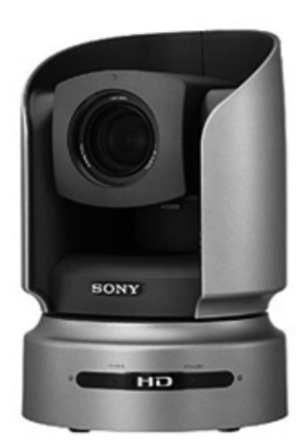

(a) BRC-H700

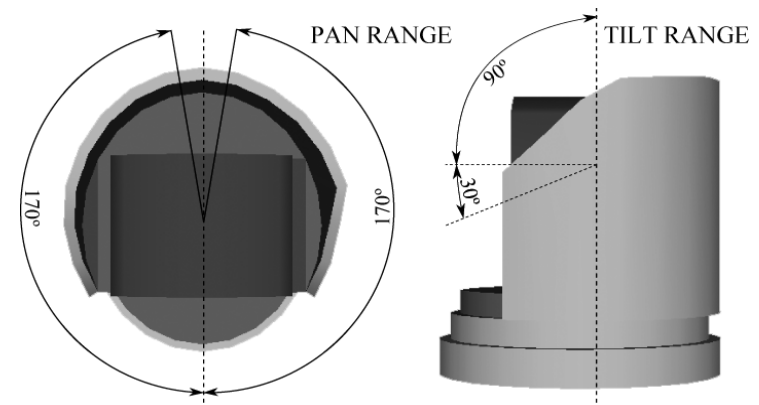

(b) BRC-H700 pan/tilt range

Fig. 5. Rotation ranges of a camera.

PTZ (pan-tilt-zoom) cameras [8], and particularly those that can be controlled remotely, are used in many fields, such as corporate training, distance learning, videoconferencing, and even broadcasting. Their main specifications are the field-of-view (FOV), image quality, and remote control distance.

The field-of-view of a camera is defined based on the rotation range of both the vertical and the horizontal axis. The rotation of the axis perpendicular to the ground is called pan (cameras can usually pan $360^{\circ}$ or slightly less; a left rotation is denoted by a negative value), and the vertical rotation is called tilt (cameras can usually tilt $180^{\circ}$; a downward rotation is denoted by a negative value). Figure 5 shows an example of these parameters for a robotic camera (Sony BRC-H700), where the pan could take values between $-170^{\circ}$ and $+170^{\circ}$ from the reference point and the tilt between $-30^{\circ}$ and $90^{\circ}$.

Other specifications of a camera are the zoom and the focus (they depend on the lens of the camera). The zoom is the adjustment of the focal length of the lens to make the target appear close-up or far away, and the focus is related to the clarity of the objects captured in the image. Usually the cameras have AutoFocus, which means that the camera is able to adjust the lens so that the target is always in focus.

This kind of robotic cameras are remotely controlled and their remote control units are usually connected to them (or other devices) by a serial port, for instance to an optical multiplex unit using optical fiber. Depending on the specific remote control unit, it is possible to program different configurations for several cameras. These configurations can store values for the pan, tilt, zoom, and focus.

The integration of the cameras in the workflow of a live broadcasting must be supported by camera operators, who need to invest an important effort to manage the cameras and keep their concentration 


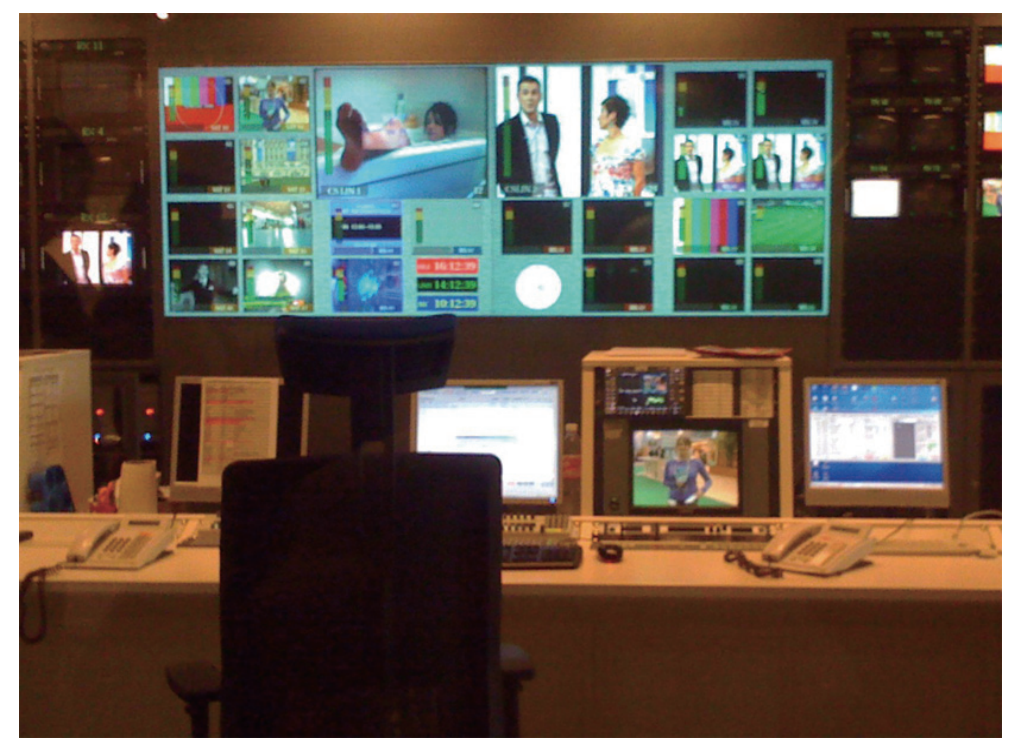

Fig. 6. Production-control room at the EiTB TV station.

continually. In many cases, according to Vicomtech's experience (http://www.vicomtech.es/), this is a very important problem, especially when the number of cameras increases significantly. In this sense, the work presented in this paper aims to provide a tool that simplifies the management of such cameras, in order to enable the production of innovative and attractive content using wireless (static or mobile) cameras controlled remotely.

\subsection{The work of the technical director}

In television, a fundamental aspect of the job of a technical director is to manually select between the available video sources, perform live edits, and overlay text on the images when it is necessary. Figure 6 shows an example of the workspace of a technical director, called Production Control Room (PCR). There are several facilities in a PCR, such as:

- A video monitor wall, where multiple sources are displayed, such as cameras, video tape recorders (VTR), graphics, and other video sources.

- A vision mixer, which is a control panel used to select the video sources that will be broadcasted or to manipulate them (e.g., adding digital effects).

- Intercom and interruptible feedback (IFB), used to communicate with crew and camera operators.

Technical directors have overall responsibility for the operations in a PCR. They coordinate the work of the whole crew, select the video source that will be on air, and look into technical problems. Technical directors have to make quick decisions in live broadcast scenarios such as the one described in this paper.

\section{Monitoring proposal}

As the underlying architecture for a location-aware system for monitoring sport events, we advocate the adoption of a general-purpose location-dependent query processing system [19], that will be extended 


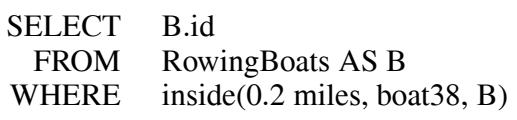

Fig. 7. Sample location-dependent query.

with the needed new functionalities. In order to get a better understanding of that system, in this section we present some basic concepts related to it. So, we first define and explain the concept of locationdependent query. Then, we summarize the general architecture proposed for location-dependent query processing. Finally, we indicate the new functionalities added to this system to manage references to videocameras in location-dependent queries.

\subsection{Location-dependent queries}

Location-dependent queries are queries whose answer depends on the locations of the objects involved. For example, a user with a smartphone may want to locate available taxi cabs that are near him/her while he/she is walking home in a rainy day. These queries are usually considered as continuous queries [42], whose answer must be continuously refreshed. For example, the answer to the previous query can change immediately due to the movements of the user and the taxi cabs. Moreover, even if the set of taxis satisfying the query condition does not change, their locations and distances to the user could change continuously, and therefore the answer to the query must be updated with the new location data.

To express location-dependent queries, we will use an SQL-like syntax with the following structure:

$$
\begin{aligned}
\text { SELECT } & \text { projections } \\
\text { FROM } & \text { sets-of-objects } \\
\text { WHERE } & \text { boolean-conditions } \\
\text { [ ORDER BY } & \text { sorting-criteria ] }
\end{aligned}
$$

where projections is the list of attributes that we want to retrieve from the selected objects, sets-of-objects is a list of object classes that identify the kind of objects interesting for the query, boolean-conditions is a boolean expression containing objects from set-of-objects that must satisfy the specified locationdependent constraints, and sorting-criteria is the ordering criteria that will be used for presentation of the results. The ORDER BY clause is optional, as in standard SQL. However, the sorting criteria can be particularly important when dealing with queries that retrieve cameras, as several cameras may satisfy the query constraints and some criteria is needed to show the most promising results first.

As an example of a location-dependent query, the query in Fig. 7 asks for rowing boats that are within 0.2 miles around boat 38 . This query includes an inside constraint expressed with the general syntax inside ( $r$, obj, target), which retrieves the objects of a certain target class (such objects are called target objects) within a specific distance $r$ (which is called the relevant radius) of a certain moving or static object $o b j$ (that is called the reference object). Thus, in the sample query in Fig. 7 the radius of the inside constraint is 0.2 miles, and there is one reference object (boat38) and one target class (RowingBoats).

It is important to emphasize that, although we use this SQL-like language to express the queries, higher-level facilities will be available for end users (e.g., predefined queries will be posed just by clicking on certain GUI buttons), as described in Section 6.

Moving and static objects in a scenario are not single points but have a certain geographic extension, depending on their size. Thus, objects and areas are managed in the same way (an object is characterized by a volume, which is its extent [38]). The techniques described in [18] to manage queries that take into account the $2 \mathrm{D}$ extent of the objects involved have been adapted in this paper to deal with $3 \mathrm{D}$ extents. 


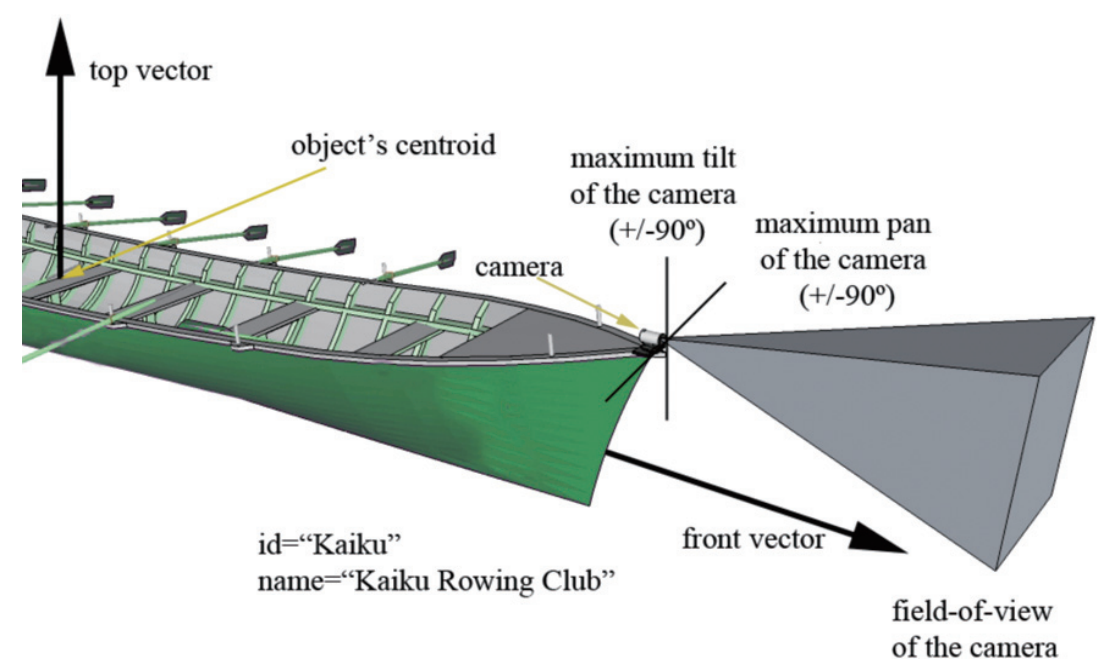

Fig. 8. Main elements of a rowing boat.

Class Objects is the set of entities in the scenario, which could be equipped with one or more cameras. RowingBoats is a subclass of Objects. An individual of Objects can be represented by the tuple:

$<i d$, name, extent, centroid, frontVector, topVector, cams $>$

where $i d$ is a unique identifier of the object, name is the name of the object, extent is the volume occupied by the object, centroid is the centroid of the extent of the object, frontVector and topVector are vectors pointing towards the frontal and the top part of the object, and cams is the list of cameras attached to the object (if any). The frontVector and topVector enable distinguishing different kinds of views of an object that could be provided by cameras (e.g., a front view of the object or a view from the top). Other interesting vectors (e.g., a vector pointing towards the bottom or the rear of the object) do not need to be defined because they can be obtained from the frontVector and topVector. For an example of the main elements characterizing a rowing boat, see Fig. 8 .

\subsection{Processing location-dependent queries}

To process location-dependent queries in a mobile environment, we have proposed in previous works the system LOQOMOTION [19] (LOcation-dependent Queries On Moving ObjecTs In mObile Networks), a distributed location-dependent query processing system whose architecture is based on mobile agents. Mobile agents $[3,43]$ are programs that execute in contexts called places, hosted on computers or other devices, and can autonomously travel from place to place resuming their execution there. Thus, they are not bound to the computer where they were created; instead, they can move freely across different computers and devices. Mobile agents provide interesting features for distributed and wireless environments (e.g., see [40]), thanks to their autonomy, adaptability, and capability to move to remote computers.

LOQOMOTION deploys a network of agents to perform the query processing over a distributed set of objects which can detect other objects moving within their range. Notice that a certain object could only detect a subset of objects in a scenario because of its limited range. The basic idea is that mobile agents move among objects in order to detect the target objects that are relevant for a query. As an example, 


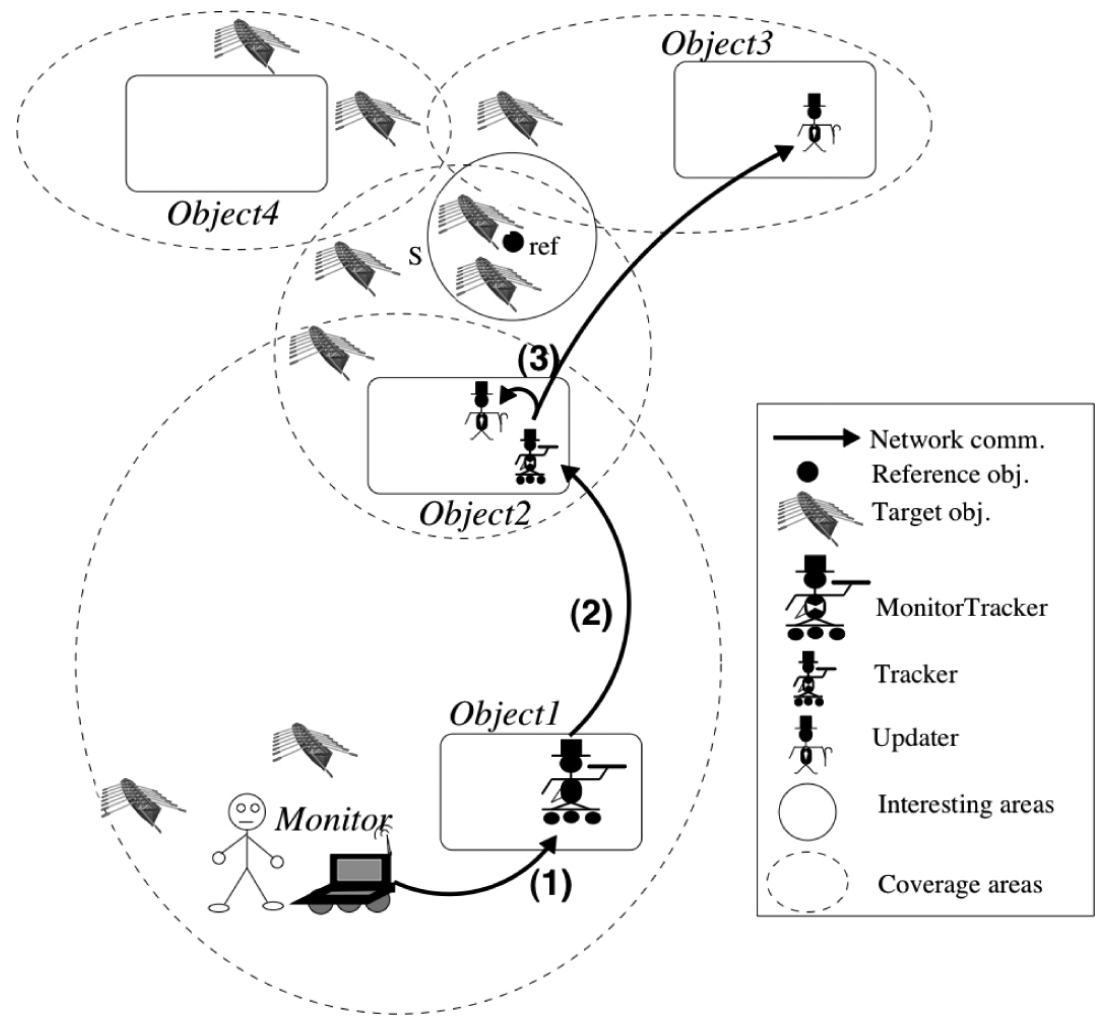

Fig. 9. Architecture of LOQOMOTION.

let us assume that in the scenario shown in Fig. 9 the object Monitor wants to retrieve the rowing boats within the area $S$ centered on the black object $r e f$.

In the figure, we represent with rectangles the objects that have the capability to detect other objects within its range (coverage area). The query will by processed by an agent called MonitorTracker, that will execute on a certain object (step 1 in Fig. 9). To do that, it first needs to know the location of ref. Notice that the object ref is beyond the coverage area of that object, and so the system will need to query some other object that is able to detect the ref object. In particular, the location of ref is known by Object2 (as ref is within its area). So, a mobile agent called Tracker travels to Object 2 to retrieve the current location of ref(step 2 in Fig. 9). Once the system knows the location of $r e f$, it also knows exactly the circular area of interest $S$. Then, the rowing boats within that area are obtained, by using agents called Updaters (step 3 in Fig. 9). Thus, one Updater executing on each object whose range intersects with $S$ (in the example, Object 2 and Object3) will keep track of the rowing boats entering $S$. Of course, as the interesting objects move, the network of agents will re-organize itself as needed (e.g., notice that when ref moves the area $S$ moves as well). For more details, see [19,20].

\subsection{Extension to monitor multimedia data: Management of videocameras}

In our context, one of the most interesting attributes of the objects in the scenario are obviously the videocameras, which play a key role for us. Thus, we do not only need to retrieve the objects that satisfy certain location-dependent constraints, but also filter out those objects whose cameras do not satisfy 


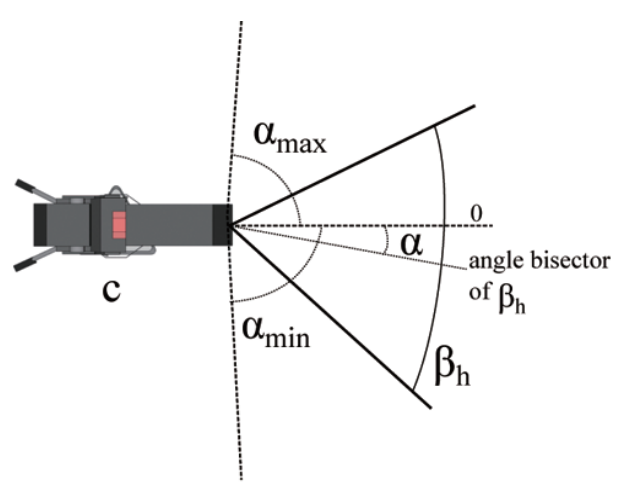

(a) pan plane

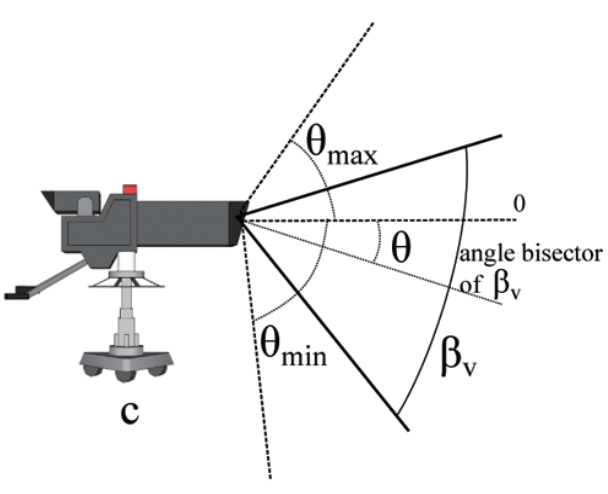

(b) tilt plane

$c=<i d, \alpha, \alpha_{\text {max }}, \alpha_{\text {min }}, \alpha_{\text {speed }}, \theta, \theta_{\text {max }}, \theta_{\text {min }}, \theta_{\text {speed }}, \beta_{h}, \beta_{v}>$

Fig. 10. Modeling a videocamera.

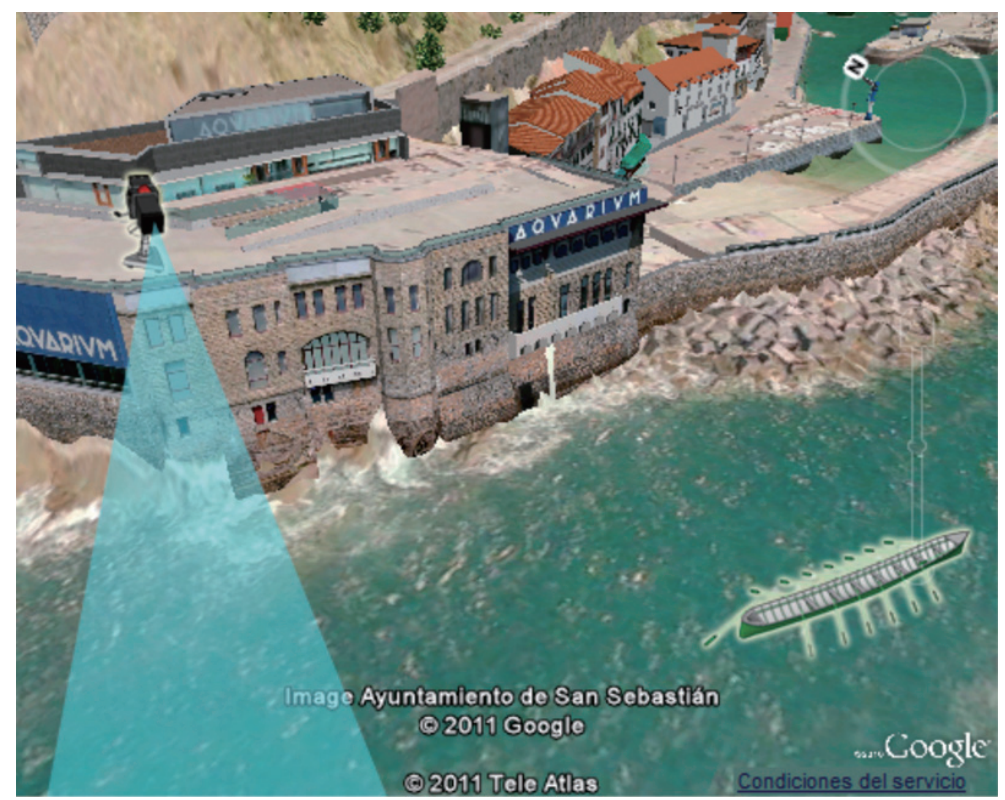

Fig. 11. 3D representation of a camera and a rowing boat.

certain conditions/features needed for a suitable viewing of the target (i.e., a static or moving object or area). For this purpose, we model a camera $c$ as shown in Fig. 10.

In the figure, $i d$ is a unique identifier, $\beta_{h}$ and $\beta_{v}$ are the horizontal and vertical angle of view, respectively. Concerning the horizontal turn (pan), $\alpha, \alpha_{\max }, \alpha_{\min }$, and $\alpha_{\text {speed }}$ are the current pan, the maximum pan possible, the minimum pan possible, and the pan speed (degrees/second) of such a camera, respectively. Concerning the vertical turn (tilt), $\theta, \theta_{\max }, \theta_{\min }$, and $\theta_{\text {speed }}$ are the current tilt, the maximum tilt possible, the minimum tilt possible, and the tilt speed (degrees/second), respectively. Thus, by considering both the pan and the tilt of a camera, the system is able to manage a 3D space containing 3D objects (Fig. 11 shows a 3D representation of a scene).

We define some functions that abstract us from the specific calculations needed, based on the features 


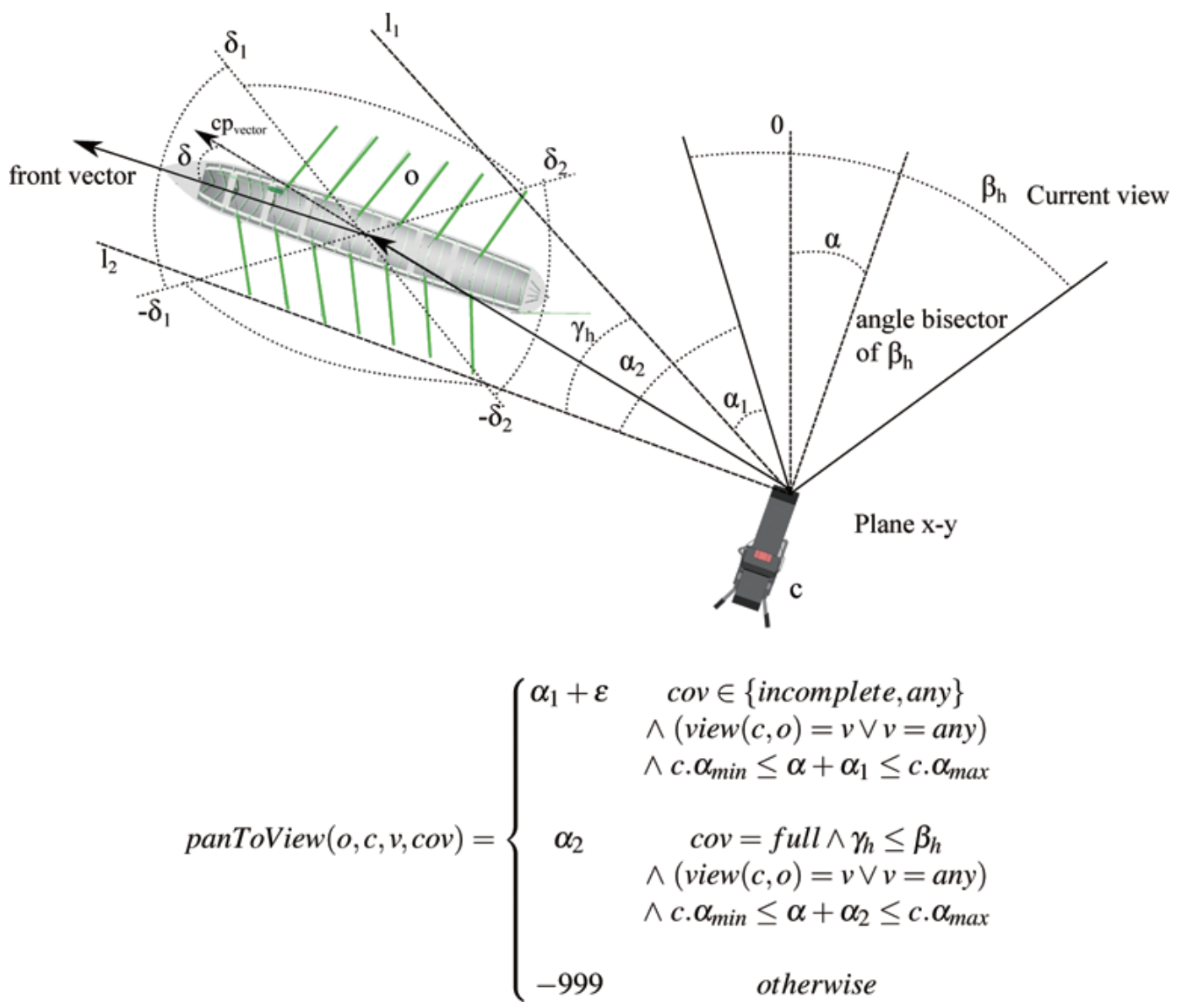

Fig. 12. Definition of the panToView function.

of the cameras, to verify certain conditions. Particularly, we define the functions panToView (see Fig. 12) and tiltToView (see Fig. 13).

These functions return the signed angle that the videocamera $c$ should pan or tilt, respectively, to view an object $o$ from a certain view $v$ (front, rear, side, top, bottom, middle, or any) with the specified coverage cov (full, incomplete, any). Positive values for panToView mean "right pan" and negative ones mean "left pan". Similarly, for tiltToView positive values mean "upward tilt" and negative ones mean "downward tilt". The sign of the angles $\alpha, \alpha_{1}, \alpha_{2}, \theta, \theta_{1}$, and $\theta_{2}$, also indicate the direction of the corresponding rotation. Notice that sometimes we would need to do both movements (pan and tilt) to be able to view an object, due to the fact that the camera and the object could be at different heights and horizontal locations. To compute the panToView, lines $l 1$ and $l 2$ (see Fig. 12) are traced from the location of the camera to both sides of the geographic volume associated to the object $o$ (in this case, a rowing boat, including its oars); similarly, lines $l 3$ and $l 4$ are traced to compute the tiltToView (see Fig. 13). It is important to consider that, as explained in Section 3.1, cameras usually have mechanical limitations about the possible turn angle ranges $\left(\left[\alpha_{\min }-\alpha_{\max }\right]\right.$ for pan and $\left[\theta_{\min }-\theta_{\max }\right]$ for tilt). So, when the camera cannot pan or tilt to view the object $o$, satisfying the specified conditions, the above functions return a special value (in our prototype, -999). In the previous formulas for panToView and tiltToView, $\varepsilon$ is a small value greater than 0 , which must be added to the angle in order to start having the target 


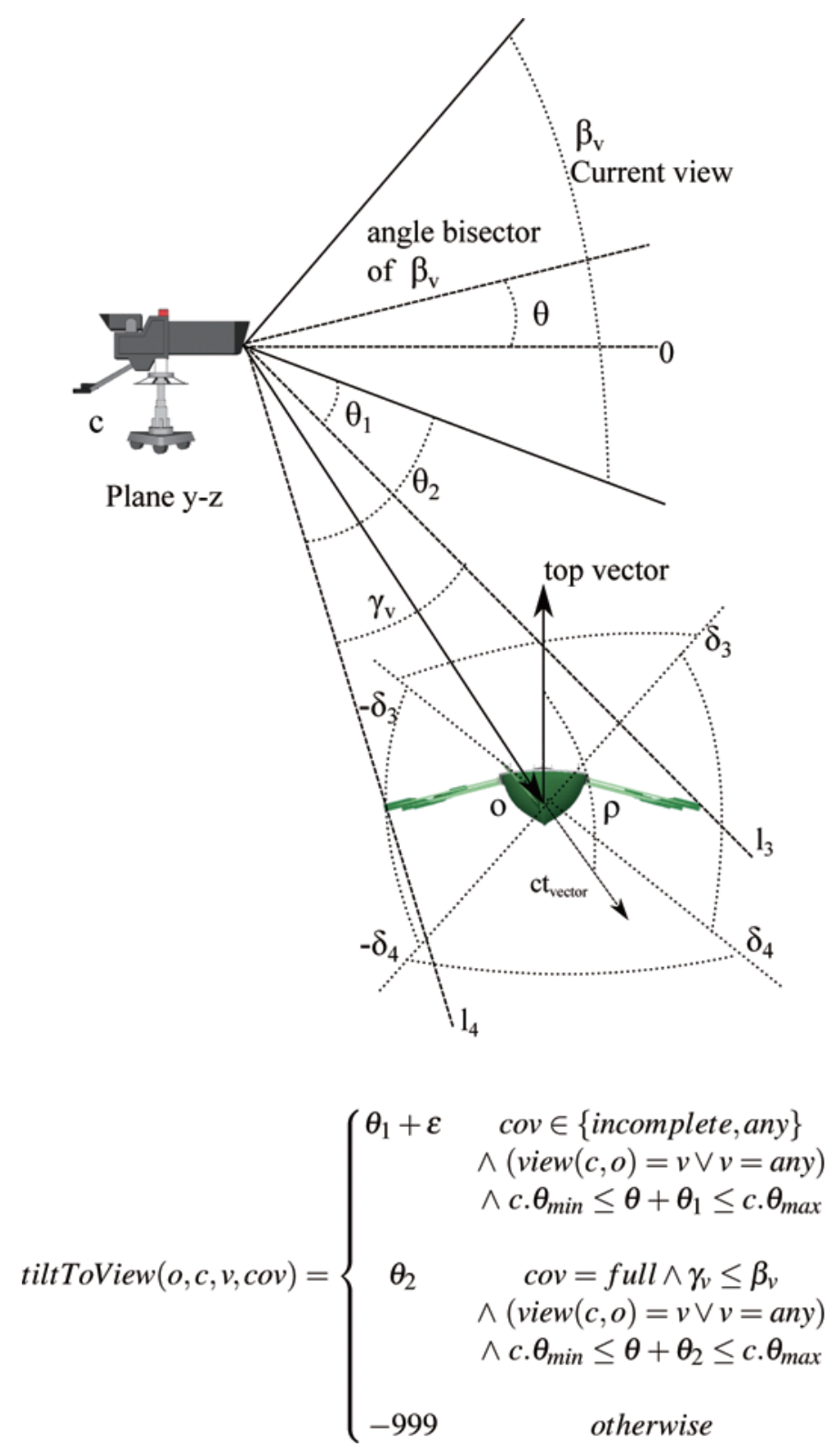

Fig. 13. Definition of the tiltToView function.

object within the view of the camera.

The function view $(c, o)$ returns values in $\{$ front, rear, side, top, bottom, middle $\}$ by considering in which of the quadrants defined by the angles $\pm \delta_{1}, \pm \delta_{2}, \pm \delta_{3}$, and $\pm \delta_{4}$ (the definition of front, rear, side, top, bottom, and middle views could be defined by any other partition) falls the angle between these two elements: 1) the vector defined by the location of the camera $c$ and the centroid (of the volume) of the target object $o$ and origin at the centroid of $o\left(c p_{\text {vector }}\right.$ in Fig. 12 and $c t_{\text {vector }}$ in Fig. 13); and 2) a predefined vector which indicates the front or the top of the object $o$, depending on whether we are considering the horizontal dimension (pan) or the vertical dimension (tilt), respectively (see angle $\delta$ in Fig. 12 and angle 
$\rho$ in Fig. 13). Notice that angles $\delta_{1}$ and $\delta_{2}$ are measured from the front vector (see Fig. 12) and angles $\delta_{3}$ and $\delta_{4}$ are measured from the top vector (see Fig. 13). Besides, angles that represent a turn to the right from the corresponding vector are considered positive and those to the left are considered negative; so, $\delta_{1}, \delta_{2}, \delta_{3}$, and $\delta_{4}$ are values that range from $0^{\circ}$ to $180^{\circ}$. The view function is defined as follows:

$$
\text { view }(c, o)= \begin{cases}\text { front } & |\delta| \geqslant \delta_{2} \\ \text { rear } & |\delta| \leqslant \delta_{1} \\ \text { side } & \left(\delta_{1}<|\delta|<\delta_{2}\right) \\ \text { top } & |\rho| \geqslant \delta_{4} \\ \text { bottom } & |\rho| \leqslant \delta_{3} \\ \text { middle } & \left(\delta_{3}<|\delta|<\delta_{4}\right) \\ & 0^{\circ} \leqslant \delta, \delta_{1}, \delta_{2}, \rho, \delta_{3}, \delta_{4} \leqslant 180^{\circ}\end{cases}
$$

Notice that view $(c, o)$ has to be applied for both the horizontal and the vertical plane. So, according to the location of the object and the camera in Figs 12 and 13, in that scenario view $(c, o)$ returns "rear" and "top". Moreover, it should be noted that if a camera must pan to focus on the target it will need some time to perform the turning. We define the function timeToPan, which returns the time (in seconds) needed to pan the camera $\alpha$ degrees:

$$
\begin{aligned}
& \operatorname{timeToPan}(o, c, v, c o v)=\frac{\text { panToView }(o, c, v, c o v)}{c \cdot \alpha_{\text {speed }}} \\
& \operatorname{timeToPan}(c, \text { pan })=\frac{\text { pan }}{c . \alpha_{\text {speed }}}
\end{aligned}
$$

Similarly, we define the timeToTilt function using tiltToView and $c . \theta_{\text {speed }}$. The cameras can pan and tilt at the same time, and therefore the time needed to pan and tilt a camera would be MAX(timeToPan, timeToTilt).

These functions will be used in the next section to take into account the features of videocameras in location-dependent queries. By defining appropriate location-dependent queries, we benefit from the power of SQL to specify in a flexible way the features of the cameras required to capture different types of views.

\section{Using LOQOMOTION in the rowing boats scenario}

In this section, we first indicate how the different elements of the query processing architecture apply in the context of rowing races in San Sebastian:

- Distributed Query Processing. In the context of rowing races in San Sebastian there is a single object (a TV trailer) that is able to access all the objects (boats, people, etc.) in the scenario. Although LOQOMOTION is particularly adapted to perform well in a distributed infrastructure where there are different objects that can monitor different geographic areas, it can obviously also work when there is a single object covering the whole area of interest.

- Inside Constraints. As cameras that are very far from their target are usually of little interest, an inside constraint with an appropriate relevant radius can be used to retrieve the candidate cameras for capturing the kind of view (close, wide open, etc.) we want (in this work we do not deal with the possibility of zooming). 


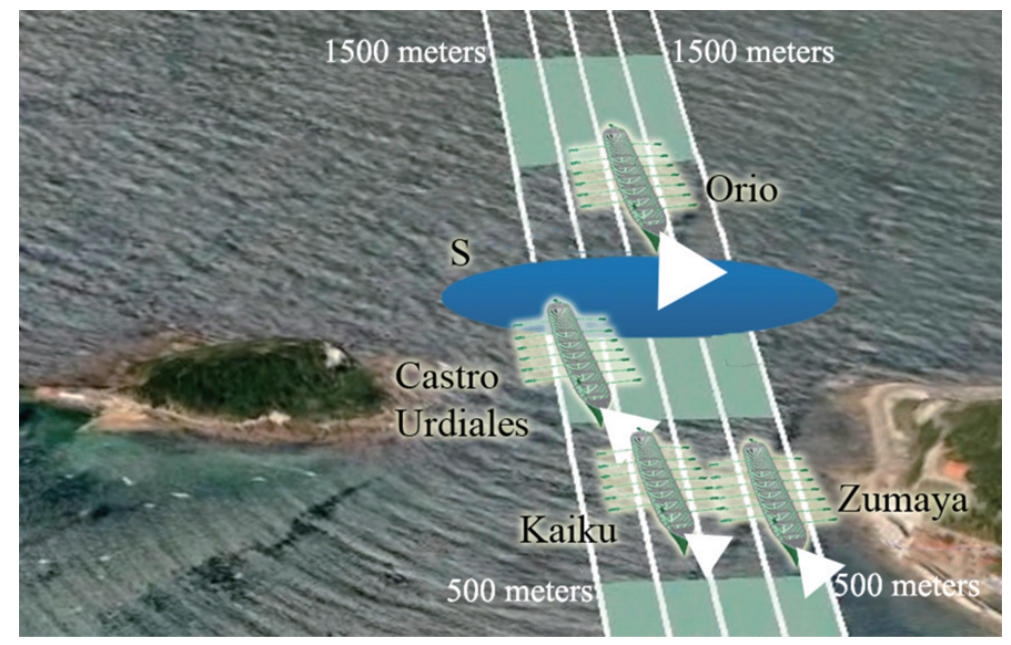

Fig. 14. Sample scenario for the example queries.

- Reference Objects. In the context of rowing races, the reference object is the interesting object (e.g., a particular boat, the island, etc.) that must be viewed. As described in Section 4.1, the extent of the objects is considered when processing the queries.

- Target Objects. In the context of rowing races, the target objects are the objects (e.g., boats) that have cameras that may satisfy the conditions required to view the area of interest. For example, cameras located on boats or fixed cameras in the harbor are part of the answer to location-dependent queries.

As seen above, the proposed architecture fits the context of rowing races in San Sebastian. In the rest of this section, we will analyze how the motivating queries described in Section 2 can be expressed (using an SQL-like syntax) in a way that allows their processing with the proposed architecture. For illustration, we will consider the scenario shown in Fig. 14 (the boats are not done to scale, as they have been enlarged for clarity), where we assume that all the boats have a camera situated in their bow, which can pan $+I-90^{\circ}$. We will use $o$.cam to reference the camera of an object $o$.

\subsection{Query 1: View a certain boat}

Let us suppose that we want to retrieve the cameras that can focus on a particular boat, for example the one named "Kaiku". The query would be expressed as follows:

SELECT O.cam.id, pan, tilt, time

FROM Objects AS O

WHERE pan=panToView(Kaiku, O.cam, any, full)

AND tilt=tiltToView(Kaiku, O.cam, any, full)

AND pan $<>-999$ AND tilt $<>-999$

ORDER BY time ASC

AND time $=$ MAX(timeToPan(O.cam, pan), timeToTilt(O.cam, tilt))

Besides the identifiers of the cameras, it returns the number of degrees that they should pan (pan value) and tilt (tilt value) to focus on the boat "Kaiku" (from any angle but providing a full view) as well as the time needed by that camera to do it (time). The cameras that currently have a full view of the "Kaiku" 


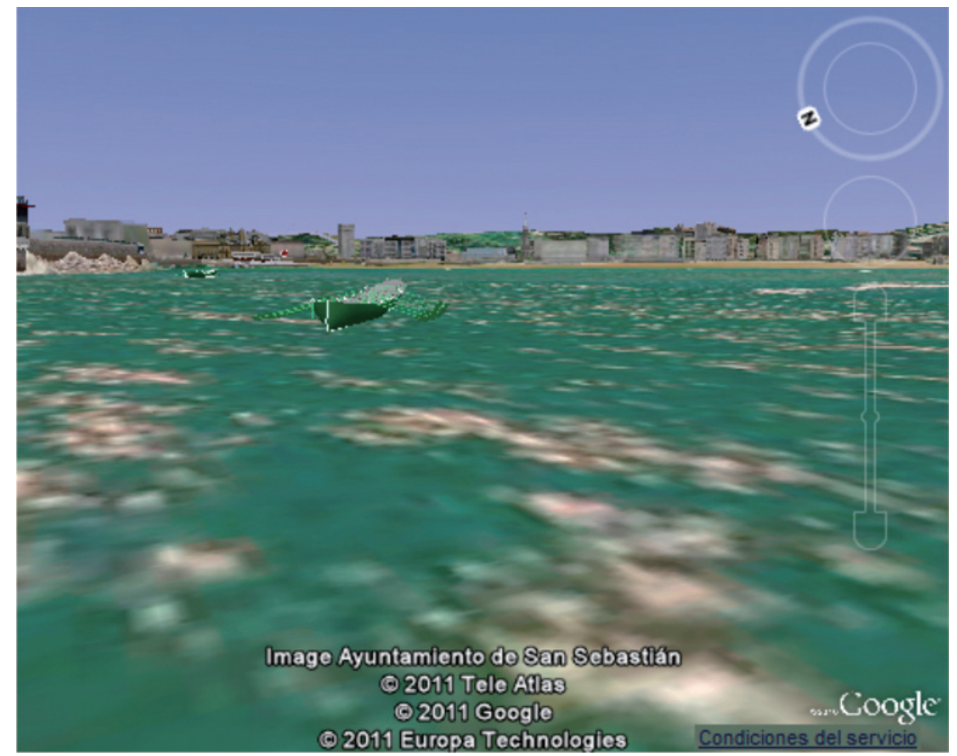

Fig. 15. Virtual view of "Kaiku" provided by "Castro Urdiales" in the scenario of Fig. 14.

boat (independently of the combination of front/rear/side and top/middle/bottom that defines the view, as for example a "front and top" view of the object) appear first in the answer ( $p a n=0$, tilt $=0$, time $=$ 0 ), and the more time they need to turn (to fully view the reference object "Kaiku") the later they appear in the answer because of the ORDER BY clause. For example, in Fig. 14 the camera of the boat "Castro Urdiales" would be retrieved first (as it has a full view of the rear of "Kaiku", see Fig. 15) and the one of "Orio" would be ranked in the second position (as it can turn slightly to have a full view of the rear of "Kaiku"). Cameras that cannot view that object (e.g., the one of the boat "Zumaya" in Fig. 14) are not included in the answer because of the pan $<>-999$ and tilt $<>-999$ constraints (see Section 4.3). However, as this query is evaluated continuously, the answer is always up to date. Thus, the information retrieved by the continuous query will help technical directors to select the best views.

\subsection{Query 2: Capture a close side view of any boat}

Let us imagine that we want to retrieve only cameras which are currently streaming a side view of a boat captured from a nearby location. This query would be expressed as follows:

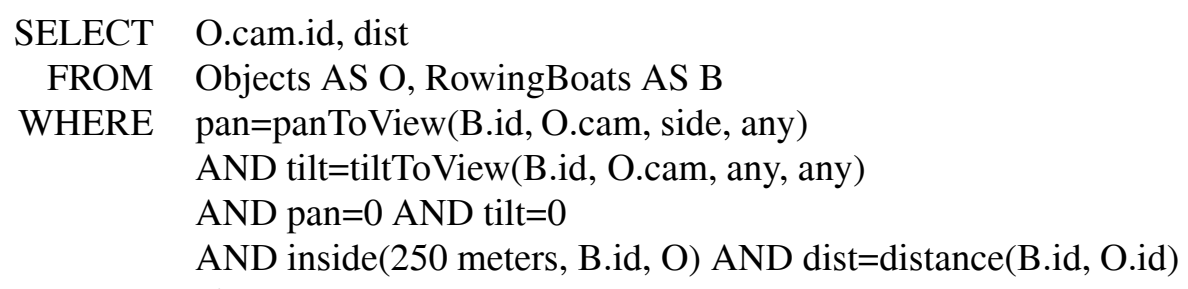

\section{ORDER BY dist}

where we assume that a camera must be located no further than 250 meters in order to provide a close (full or incomplete) view of that boat. Moreover, we order the cameras according to the distance to the target, as closer cameras are more likely to be able to provide detailed views. Thus, for example, in the 


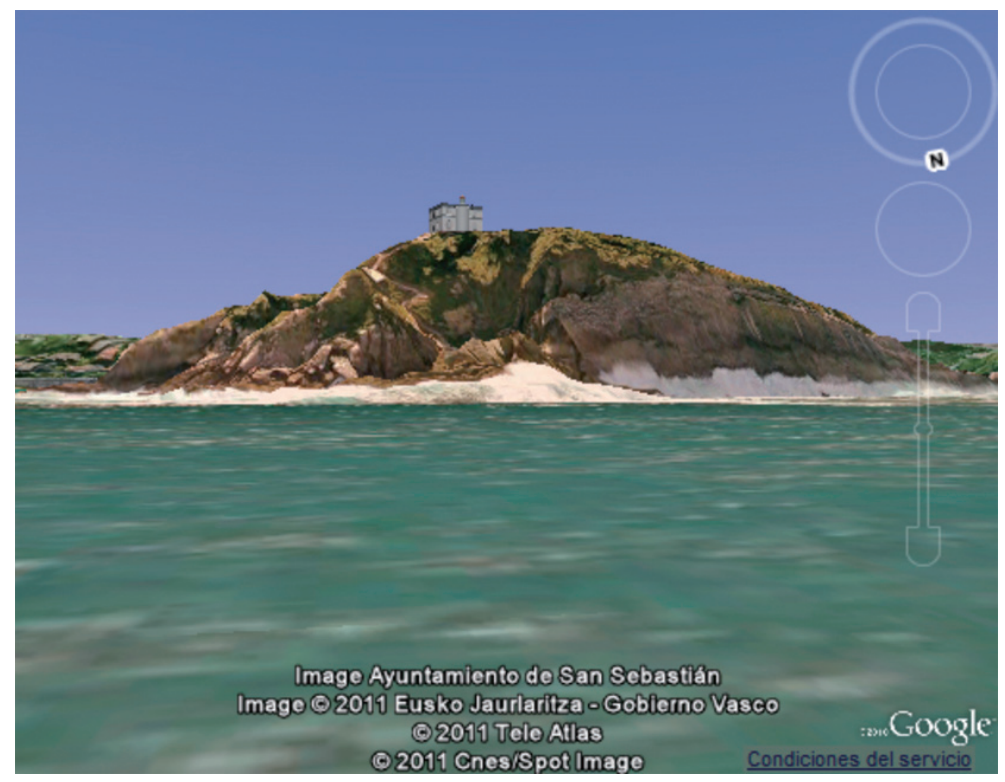

Fig. 16. Virtual view of the island from the ocean side.

scenario of Fig. 14 the camera of "Castro Urdiales" would be a good candidate to view the boat "Kaiku" (see Fig. 15).

\subsection{Query 3: Capture a wide view of the island from the ocean side}

For this query, let us assume that the technical director previously defined an area $S$ (see Fig. 14), on the ocean side, which represents where a camera should be located in order to have the wanted wide view of the island from the ocean side (see Fig. 16). Thus, the query could be expressed as follows:

SELECT O.cam.id, pan, tilt, time

FROM Objects AS O

WHERE inside(0 meters, S, O)

AND pan=panToView(island, O.cam, any, full)

AND tilt=tiltToView(island, O.cam, any, full)

AND pan $<>-999$ AND tilt $<>-999$

AND time $=$ MAX (timeToPan(O.cam, pan), timeToTilt(O.cam, tilt))

ORDER BY time ASC

where with the constraint inside $(0$ meters, $S, O)$ we require objects no further than 0 meters from $S$ (that is, objects within $S$ ). In the scenario shown in Fig. 14 no camera is currently within the area $S$. However, the "Orio" boat will enter soon $S$ and so it will become part of the answer to the continuous query. It should be noted that the technical director may want to change the size and location of the area $S$ at any time, in order to focus on a different area (e.g., an accident that has happened). The system is able to adapt to changes in the monitoring requirements.

In Table 1 we summarize the current answers to the sample queries discussed in this section, according to the scenario shown in Fig. 14. 
Table 1

Answers for the sample queries

\begin{tabular}{ll}
\hline Query & Answer (cameras) \\
\hline 1.- View a certain boat & -"Castro Urdiales" \\
("Kaiku") & - -Orio" \\
2.- Capture a close side & For the "Kaiku" boat: \\
view of any boat & -"Castro Urdiales" \\
3.- Capture a wide view of & No answer \\
the island from the ocean & for the moment \\
\hline
\end{tabular}

\section{Prototype and experimental evaluation}

In order to test the system proposed, a prototype has been developed. This prototype consists of three modules: 1) a simulator, 2) a query processor, and 3) a Graphical User Interface (GUI) for technical directors. Testing this kind of system in a real-life environment is difficult because there are many real objects, devices, and wide-area scenarios involved; therefore, we have had no chance to test it on the real rowing race in San Sebastian, which is celebrated only once a year. So, the simulator of wireless and distributed environments described in [21] has been adapted to deal with the sample scenario of this paper. This simulator used a file containing the real GPS location data of each rowing boat recorded every second during the rowing race celebrated in September 2010. Therefore, real trajectories were used to move objects in the simulator. Moreover, the simulator allows us to dynamically change other parameters, such as the current pan and tilt of each camera, to simulate the real movements of the cameras.

The technical director needs a simple GUI that simulates his/her work environment, where the query processor is integrated. As we are not using any real camera in the test, cameras are simulated using the Google Earth API (http://code.google.com/apis/earth/), to show an approximate view of what the cameras would view in the real scenario. This was inspired by the work presented in [28], where the data coming from GPS receivers in the boats during the broadcasting of live rowing events are integrated into a 3D terrain model. Figure 17 shows a screenshot of this GUI in our prototype. The technical director could see up to four camera streams at the same time and use the buttons on the bottom left panel to interact with the query processor, as explained in Section 5. This interface is inspired by mobile production units where the technical director has several input video sources to select, a screen where the source selected is processed (Preview screen), and another screen (Program screen) to see what it is being broadcasted; in our GUI, by clicking on the button "air", we "broadcast" the content of the Preview screen into the Program screen.

The system has been tested using the query described in Section 5.1. In that query, the technical director wants to retrieve all the cameras that could provide a full coverage and any view of the rowing boat "Kaiku". The parameters used in the tests are the following ones:

- There are four rowing boats with a camera each; they are moving according to the real GPS location information captured during the race in 2010.

- There are three fixed cameras, one on the island, another one on the promenade, and the last one on a sailing boat near the rowing boats.

- The cameras are set with horizontal focus $70^{\circ}$, vertical focus $45^{\circ}$, pan range $\pm 130^{\circ}$, tilt range $\pm 90^{\circ}$, pan and tilt speed 5.5 degrees/second.

In the rest of this section, we evaluate the precision and recall of the system, as well as the precision of the estimation of the time needed by a camera to focus on a target. 


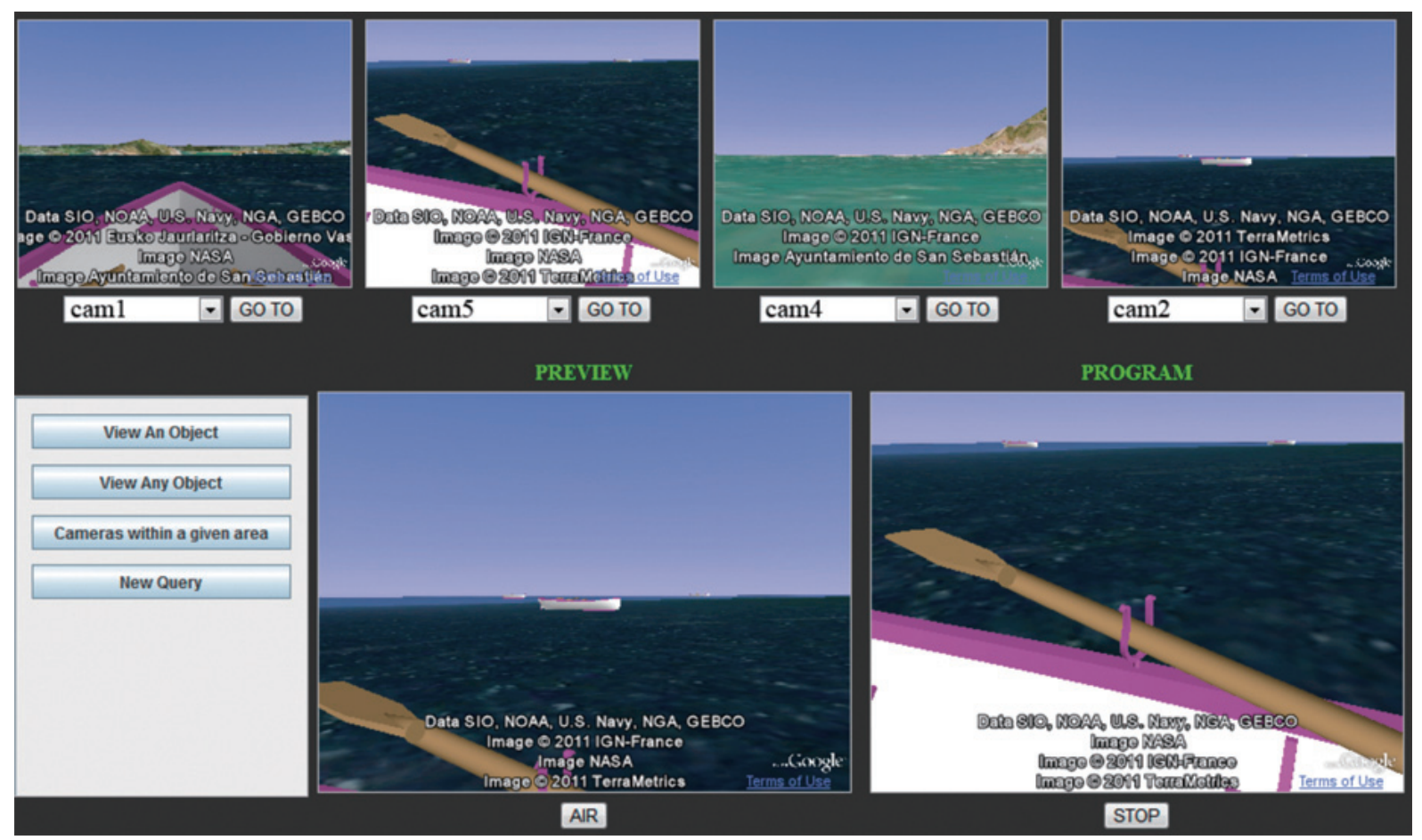

Fig. 17. GUI for the technical director.

\subsection{Testing the precision and recall of the cameras in the answer}

The first test is conceived to obtain the difference between the number of cameras in an answer given by the system in real time and the optimal answer. Figure 18 shows the results of this test, where we are representing the number of cameras given in the answer (which is refreshed every second). Remember that there exist seven cameras in the scenario. An error is indicated in the graph with an arrow at the specific time instant when such an error occurs. When an answer includes, for example, one extra camera (a camera that will not obtain the required view), it is denoted by "+1 Cam" and when the answer misses one valid camera (one that could obtain the required view), it is denoted by "-1 Cam". Along with the number of extra/missing cameras, we specify between brackets the amount of time (in seconds) that this error lasts.

During the race, the graph shows that the coverage of "Kaiku" is quite high compared with the number of existing cameras: the maximum number is six (because the camera of "Kaiku" obviously cannot fully focus on itself) and at least three cameras obtain the required view on the target rowing boat. Notice also that the errors committed by the system never last more than 2 seconds. The reason is the efficient continuous refreshment of the answer.

It can also be observed that the number of cameras able to capture the target varies quickly once the turning point is passed (around time=10:00). This variation is due to the query asking for a full view of "Kaiku". As the distance and speed of the rowing boats increase, some cameras are unable to maintain the target completely focused all the time (this would require exceeding the pan limit), and so they disappear for a short time from the answer set even when they can provide a view that covers most of the target object. 


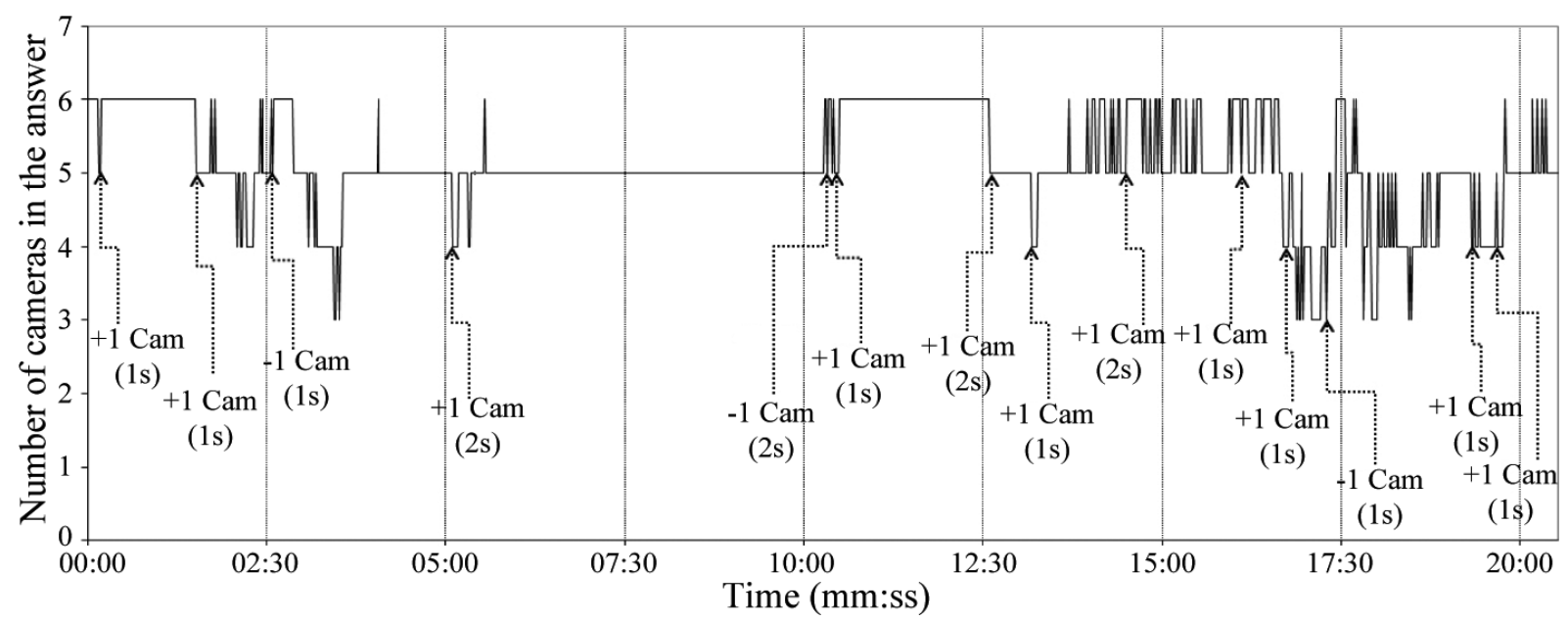

Fig. 18. Error in precision/recall of the cameras.

Even when the query processor must perform its calculations every second, the results are quite satisfactory for a period of twenty minutes, and the errors are corrected quickly enough to not be taken into account by the user, i.e., the technical director.

\subsection{Testing the precision of the estimated time to view}

The goal of the second test is to evaluate the precision of the answer given by the query processor in terms of the time information provided. When the query processor lists a camera in the answer, the time needed by the camera to turn (pan and/or tilt) to focus on the rowing boat is computed and shown to the technical director. This estimation could be wrong because the rowing boats, and therefore their cameras, could move unexpectedly, although these errors will be corrected quickly as the query is continuously processed.

In Fig. 19, we show the total error committed by all the cameras (the difference between the time given by the query processor to turn the cameras and the real time needed) at every instant. In the figure, we use triangles to mark time instants when a camera is included in the answer but its error is too big (i.e., that camera should not be included in the answer).

Initially, all the cameras (pointing at the same direction as the front vector of their rowing boats) have to pan to focus on "Kaiku" and the query processor estimates the time needed for the movement with a small error. After that, the error is zero because the rowing boats are moving at approximately the same speed. Around time 02:30, the rowing boats are leaving the bay and they get closer to each other. "Kaiku" is too close to its neighbor boat whose camera onboard is not able to fully focus on "Kaiku". The next interesting time instant is around 10:00, when the rowing boats are in the turning point. Here the error is caused by the neighbor boat of "Kaiku" because the system estimates that the time to view "Kaiku" is much greater than the real time needed, due to the quick turning maneuver. Once the turning point is passed, the speed of the rowing boats and their relative distance increase. That means that the fastest rowing boats are not going to be able to focus on "Kaiku" during a few seconds (around time 14:00). Finally, the maximum distance between the rowing boats is achieved, and so there are some cameras that cannot view "Kaiku" anymore and the system incurs several errors, indicated by the triangles around time 17:30-20:00. 


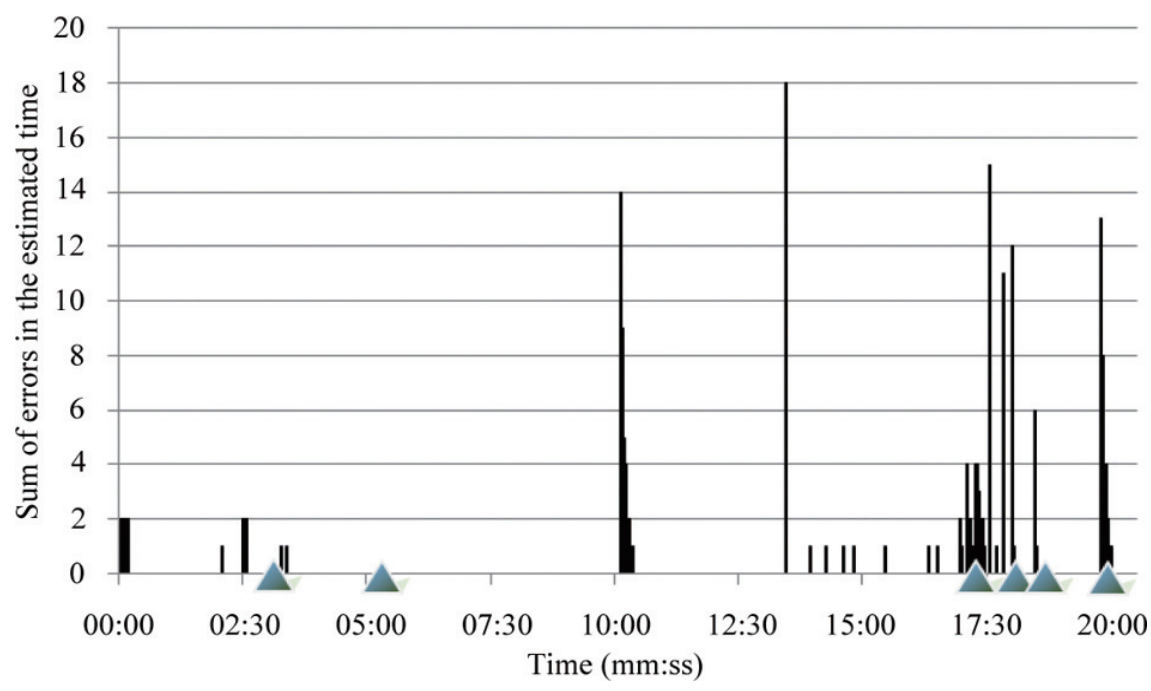

Fig. 19. Error in the estimated time.

This test shows that the system commits small errors concerning the estimated time to view the target. Besides, those errors are fixed very quickly (in the test, in five seconds at the most), since the queries are continuously processed. The answer presented to the user contains too high errors only six times along the twenty minutes of race (remember that an answer is updated every second), and those errors only last one second.

\section{Related work}

In this section, we analyze works that focus on the use of multiple cameras to produce contents (for video lectures, sport events, and others) as well as works that are closer to the field of locationbased services. Works performing object tracking by using multiple cameras [24,50] are not explicitly considered, as they pursue a different goal: estimating/monitoring the trajectory of an object from images, and in our proposal the objects are detected by using sensors [30] (specifically, GPS receivers).

\subsection{Camera management for video lectures}

Several works in the literature have considered the problem of automatic camera management for recording and broadcasting lectures, talks and seminars [4,5,17,29-31,39,41,51]. In this section, we provide an overview of some proposals:

- One of the first works in the area is the AutoAuditorium system [4,5] (see http://www.autoauditorium. $\mathrm{com} /$ ), which uses two cameras and microphones to obtain information about what is happening on the stage and perform an automatic audio mixing, tracking of the people on stage, and automatic camera selection.

- Another interesting work is [30,39], which also proposes a two-camera system that is able to track the presenter and the audience and generates automatically the produced video. Several production rules have been implemented in this system, inspired by the way professional video producers work, in order to take the appropriate recording decisions. According to the tests performed, the system passes the Turing test successfully. 
- The system FlySPEC, proposed in [29], combines a PTZ camera and a panoramic camera and integrates requests from users, along with a mechanism to solve conflicting requests. In this way, the system benefits from the involvement of the audience to reduce the probability of unsatisfactory recordings.

- As another example [51], presents the Microsoft Research LeeCasting System (MSRLCS), that supports a scripting language to facilitate the customization of production rules for different room configurations and production styles.

- The Virtual Director described in [31] considers an automation specification language (ASL) that directors can use to express broadcast specifications.

Finally, it is interesting to mention the Virtual Videography approach described in [17]. Whereas the previous works perform a live broadcast, this last work advocates an offline processing, as this provides more time and more information to perform the video production (e.g., it is possible to look into the future).

Although the context and purpose of these works is different than the ones considered in this paper, they highlight the interest of the development of automatic video production techniques to save production costs and enable fast access to multimedia information.

\subsection{Camera management for sport events}

Several other works have focused on helping producers of sport videos [34], as we describe in this section.

Some of these works $[6,7,12]$ are part of the APIDIS (Autonomous Production of Images based on Distributed and Intelligent Sensing) project (http://www.apidis.org/). For example, in [7] a system that helps the video production and video summarization in the context of basketball games is presented. This system is based on a divide-and-conquer strategy and considers the trade-off among three desirable properties: closeness (resolution or level of detail of the images), smoothness (continuity in the movement and in the story-telling contents, despite the switching of camera views), and completeness (displaying as many objects and interesting actions as possible). Besides, user preferences are taken into account in order to provide personalized videos.

Outside the APIDIS project, the My eDirector 2012 project, presented for example in [36], advocates extracting semantic metadata from the video streams to enable the personalization of the multimedia information broadcasted. Several problems are considered in this project, such as the detection and tracking of athletes and the automatic detection and tracking of high-level patterns. For more information, the web page of the project can be consulted (http://www.myedirector2012.eu/).

Besides basketball and athletic competitions, producing suitable soccer videos has also attracted the interest of research. As an example, the work presented in [14] tackles the problem of summarizing videos (i.e., obtaining video abstracts) of soccer games by applying different image processing algorithms to analyze the input videos. Soccer videos were also considered as an evaluation scenario within the context of the APIDIS project (see [6]). The work in [2] also focuses on the analysis and summarization of soccer videos by using image recognition techniques and presents an experimental evaluation based on criteria such as the video quality, the intelligibility, the quality of the zooming and panning performed, the view size, and the view duration. As another example, a multi-camera tracking approach based on a belief propagation algorithm is proposed in [13], where thanks to the collaboration among the cameras it is possible to compensate the effects of poor observations due to phenomena such as occlusions. There are other works and patents that deal with the problem of summarizing soccer videos, such as [35]. 
Other sports have also been considered, such as tennis, baseball, and cricket. For example, in the TennisSense platform [9], nine IP cameras around the tennis court are used to automatically infer what is happening in a tennis game. The extracted metadata is then used for indexing and browsing the stored tennis videos. In [33] the problem of video summarization is considered in the context of baseball games. The proposed method is able to generate abstracts based on metadata describing the semantic content of videos encoded in the MPEG-7 format. Moreover, it takes into account the preferences of the users to build a personalized summary of the input video. Finally, in [26] excitement clips (e.g., the close-up of a player or the referee, the gathering of players, etc.) from sports videos are extracted by exploiting audio features (such as an increase in the audio level of the voice of the commentators or the cheers of the audience). The proposal is validated by using soccer and cricket videos.

All the works described in this section tackle sport events, like the proposal in this paper, but they have a different purpose. Thus, our system helps video producers to locate cameras that can provide certain images in an environment with moving objects and moving cameras, whereas the purpose of these works is usually to perform an automatic video production in an offline setting (so, for example, achieving a good performance for real-time processing is not an issue) by using image processing techniques. In other words, the focus of these works is on the operation and exploitation of the cameras, rather than on the complexity of managing multiple video signals in real time.

Although some works explicitly mention the possibility of live broadcasting, such as [47] (that focuses on soccer games and indicates that even though their system is currently performing off-line it can be improved to enable on-line processing), the context of these works is different from the one presented in this paper. Thus, for example, moving cameras are not used in those related works. However, according to [47], the automatic generation of broadcast video is facilitated when a fixed set of static cameras is considered, since the types of views available (e.g., far view, close-up view, etc.) is limited by the set of cameras used. According to [11], which presents a multi-camera selection technique based on features of objects and frames and considers a basketball game scenario (among others), an efficient mechanism for detection, tracking and feature extraction is a key element to enable view selection in a short time.

\subsection{Other works on multi-camera management}

In this section we briefly indicate some other interesting works for multi-camera management. An interesting and representative work concerning the management of multiple cameras and screens can be found in [10], which proposes different approaches for concurrent access and management of multiple static cameras. It is also worth mentioning the proposal in [46], where the authors work on the optimal monitoring of multiples video signals. In [1] the authors use an array of cameras to acquire a 3D scene.

However, it is important to emphasize that these representative works on multi-camera management and monitoring consider only cameras that are static (i.e., at fixed locations). On the contrary, the cameras considered in our proposal can move.

\subsection{Works on location-dependent queries}

Developing query processing techniques that manage location-dependent queries efficiently is an intensive area of research [22,45], due to the great interest in Location-Based Services (LBS). The existing proposals differ in several aspects, such as the types of queries that they can process, the assumptions that they rely on, and/or the underlying infrastructure required. For example, MobiEyes [15] requires the cooperation of the moving objects that are the targets of the queries to process the queries distributively, LOQOMOTION [19] performs a distributed processing on certain objects, the proposal in [25] considers 
a multi-cell distributed environment to process range queries over static target objects, DOMINO [48] exploits some knowledge about the trajectories of the objects, $S E A-C N N$ [49] focuses on the processing of continuous kNN queries, the work presented in [32] considers soft timing requirements that may exist for the processing of location-dependent queries, and [44] tackles location-dependent queries in a wireless broadcast environment through a global indexing scheme.

However, we are not aware of any work that has applied an architecture for processing locationdependent queries in the context of sport events to retrieve relevant multimedia data. Although there are works that emphasize the importance of location-dependent query processing for multimedia computing (e.g., [27]), they do not consider that the queries themselves can have as a final goal to retrieve multimedia data that are relevant for the user. Works that propose location-dependent multimedia services for mobile users (e.g., [37], where a middleware based on mobile agents is presented) are not directly related either to the research presented in this paper.

Moreover, as far as we know, the processing of location-dependent queries has not been studied before in relation to any kind of multimedia data.

\section{Conclusions and future work}

In this paper, we have shown the usefulness of location-dependent query processing in a real-world sport event: the rowing races in San Sebastian. With that purpose, we have extended a general architecture for location-dependent query processing with the capabilities needed to detect suitable (static or moving) cameras to view a particular moving object or area. Besides, we have shown the precision of our system by testing our prototype using real GPS location data captured during a rowing race celebrated in September 2010. The main features of our proposal are:

- The features of videocameras are considered to help technical directors to access the multimedia information coming from different (static or moving) videocameras.

- Predefined or dynamically built geographic areas or static/moving objects can be set as targets for which suitable cameras can be found by the system.

- The proposal is flexible, and so new functionalities can be added to the system without important changes to the main architecture.

The underlying location-dependent query processing system used benefits from the use of mobile agents to carry out the processing tasks wherever they are needed. Thus, agents are in charge of 1) tracking the location of interesting (static or moving) objects and the field-of-view of all the cameras, and 2) refreshing the query answers continuously and efficiently.

As future work, we plan to extend the system to enable automatic tracking of targets by videocameras, in order to further help the technical directors with the broadcasting process. It would be interesting to extend this study to other sport scenarios too. Particularly, we think that the context of classic cycling competitions, such as the Tour of France, could be very challenging (the scenario could extend along many kilometers in mountain landscapes where a centralized solution could be unsuitable). In other scenarios, some useful multimedia information could also be provided by mobile devices carried by people (e.g., spectators or tourists), and so exploiting the possibilities of Mobile Ad Hoc Networks (MANETs) for content dissemination [16] and retrieval of multimedia information would be interesting. In these highly distributed and dynamic scenarios, the whole potential of LOQOMOTION could be better exploited. 


\section{Acknowledgments}

This research work has been supported by the CICYT project TIN2010-21387-C02. We also thank David Antón and Aritz Legarretaetxebarria for their help with the implementation of our prototype and technical support, respectively.

\section{References}

[1] D.G. Aliaga, Y. Xu and V. Popescu, Lag camera: A moving multi-camera array for scene-acquisition, Journal of Virtual Reality and Broadcasting 3(10) (December 2006).

[2] Y. Ariki, S. Kubota and M. Kumano, Automatic production system of soccer sports video by digital camera work based on situation recognition. In Eighth IEEE International Symposium on Multimedia (ISM'06), IEEE Computer Society, 2006, pages 851-860.

[3] F.L. Bellifemine, G. Caire and D. Greenwood, Developing Multi-Agent Systems with JADE, Wiley, 2007.

[4] M.H. Bianchi, AutoAuditorium: A fully automatic, multi-camera system to televise auditorium presentations. In 1998 Joint DARPA/NIST Smart Spaces Technology Workshop, 1998.

[5] M.H. Bianchi, Automatic video production of lectures using an intelligent and aware environment. In Third International Conference on Mobile and Ubiquitous Multimedia (MUM'04), ACM Press, 2004, pages 117-123.

[6] F. Chen and C.D. Vleeschouwer, A resource allocation framework for summarizing team sport videos. In 16th IEEE International Conference on Image Processing (ICIP'09), IEEE Computer Society, 2009, pages 4293-4296.

[7] F. Chen and C.D. Vleeschouwer, Personalized production of basketball videos from multi-sensored data under limited display resolution, Computer Vision and Image Understanding 114(6) (June 2010), 667-680.

[8] I.-H. Chen and S.-J. Wang, An efficient approach for the calibration of multiple PTZ cameras, IEEE Transactions on Automation Science and Engineering 4(2) (April 2007), 286-293.

[9] C.O. Conaire, P. Kelly, D. Connaghan and N.E. O'Connor, TennisSense: A platform for extracting semantic information from multi-camera tennis data. In 16th International Conference on Digital Signal Processing (DSP'09), IEEE Computer Society, 2009, pages 1062-1067.

[10] G.W. Daniel and M. Chen, Interaction control protocols for distributed multi-user multi-camera environments, Systemics, Cybernetics and Informatics 1(5) (2003), 29-38.

[11] F. Daniyal, M. Taj and A. Cavallaro, Content and task-based view selection from multiple video streams, Multimedia Systems 46(2-3) (January 2010), 235-258.

[12] D. Delannay, N. Danhier and C.D. Vleeschouwer, Detection and recognition of sports(wo)men from multiple views. In Third ACM/IEEE International Conference on Distributed Smart Cameras (ICDSC'09). IEEE Computer Society, 2009.

[13] W. Du, J. bernard Hayet, J. Piater and J. Verly, Collaborative multi-camera tracking of athletes in team sports. In Workshop on Computer Vision Based Analysis in Sport Environments (CVBASE'06), 2006, pages 2-13.

[14] A. Ekin, A.M. Tekalp and R. Mehrotra, Automatic soccer video analysis and summarization, IEEE Transactions on Image Processing 12(7) (July 2003), 796-807.

[15] B. Gedik and L. Liu,. MobiEyes: A distributed location monitoring service using moving location queries, IEEE Transactions on Mobile Computing 5(10) (October 2006), 1384-1402.

[16] J. Haillot and F. Guidec, A protocol for content-based communication in disconnected mobile ad hoc networks, Mobile Information Systems 6(2) (April 2010), 123-154.

[17] R. Heck, M. Wallick and M. Gleicher, Virtual videography, ACM Transactions on Multimedia Computing, Communications, and Applications 3(1) (February 2007), 4:1-4:28.

[18] S. Ilarri, C. Bobed and E. Mena, An approach to process continuous location-dependent queries on moving objects with support for location granules, Journal of Systems and Software 84(8) (August 2011), 1327-1350.

[19] S. Ilarri, E. Mena and A. Illarramendi, Location-dependent queries in mobile contexts: Distributed processing using mobile agents, IEEE Transactions on Mobile Computing 5(8) (August 2006), 1029-1043.

[20] S. Ilarri, E. Mena and A. Illarramendi, Using cooperative mobile agents to monitor distributed and dynamic environments, Information Sciences 178(9) (May 2008), 2105-2127.

[21] S. Ilarri, E. Mena and A. Illarramendi, A system based on mobile agents to test mobile computing applications, Journal of Network and Computer Applications 32(4) (July 2009), 846-865.

[22] S. Ilarri, E. Mena and A. Illarramendi, Location-dependent query processing: Where we are and where we are heading, ACM Computing Surveys 42(3) (March 2010), 1-73.

[23] S. Ilarri, E. Mena, A. Illarramendi and G. Marcos, A location-aware system for monitoring sport events. In Eight International Conference on Advances in Mobile Computing \& Multimedia (MoMM 2010), ACM, Austrian Computer Society (OCG), 2010, pages 305-312. 
[24] O. Javed and M. Shah, Automated Multi-Camera Surveillance: Algorithms and Practice, volume 10 of The Kluwer International Series in Video Computing, chapter 5 "Tracking in Multiple Cameras with Disjoint Views", Springer, 2008, pages 59-84.

[25] J. Jayaputera and D. Taniar, Data retrieval for location-dependent queries in a multi-cell wireless environment, Mobile Information Systems 1(2) (April 2005), 91-108.

[26] M.H. Kolekar, Bayesian belief network based broadcast sports video indexing, Multimedia Tools and Applications 54(1) (August 2011), 27-54.

[27] A. Krikelis, Location-dependent multimedia computing, IEEE Concurrency 7(2) (April/June 1999), 13-15.

[28] M. Laka, I. García, I. Macía and A. Ugarte, TV sport broadcasts: Real time virtual representation in 3D terrain models. In 3DTV Conference: The True Vision - Capture, Transmission and Display of 3D Video (3DTV-Con'08), IEEE Computer Society, 2008, pages 405-408.

[29] Q. Liu, D. Kimber, J. Foote, L. Wilcox and J. Boreczky, FlySPEC: a multi-user video camera system with hybrid human and automatic control. In Tenth ACM International Conference on Multimedia (MULTIMEDIA'02), ACM Press, 2002, pages 484-492.

[30] Q. Liu, Y. Rui, A. Gupta and J.J. Cadiz, Automating camera management for lecture room environments. In SIGCHI Conference on Human Factors in Computing Systems (CHI'01), ACM Press, 2001, pages 442-449.

[31] E. Machnicki and L.A. Rowe, Virtual director: automating a webcast. In Multimedia Computing and Networking 4673, SPIE, 2002, pages 208-225.

[32] Z. Mammeri, F. Morvan, A. Hameurlain and N. Marsit, Location-dependent query processing under soft real-time constraints, Mobile Information Systems 5(3) (August 2009), 205-232.

[33] N. Nitta, Y. Takahashi and N. Babaguchi, Automatic personalized video abstraction for sports videos using metadata, Multimedia Tools and Applications 41(1) (January 2009), 1-25.

[34] J. Owens, Television Sports Production. Focal Press, 2007. Fourth Edition.

[35] H. Pan and B. Li. Summarization of soccer video content. United States Patent 7657836, 2004.

[36] N. Papaoulakis, N. Doulamis, C. Patrikakis, J. Soldatos, A. Pnevmatikakis and E. Protonotarios, Real-time video analysis and personalized media streaming environments for large scale athletic events. In First ACM Workshop on Analysis and Retrieval of Events/Actions and Workflows in Video Streams (AREA'08), ACM Press, 2008, pages 105-112.

[37] M.H. Raza and M.A. Shibli, Mobile agent middleware for multimedia services. In Ninth International Conference on Advanced Communication Technology (ICACT'07), IEEE Computer Society, 2007, pages 1109-1114.

[38] P. Rigaux, M. Scholl and A. Voisard, Spatial databases with application to GIS, Morgan Kaufmann Publishers Inc., 2002.

[39] Y. Rui, L. He, A. Gupta and Q. Liu, Building an intelligent camera management system. In Ninth ACM International Conference on Multimedia (MULTIMEDIA'01), ACM Press, 2001, pages 2-11.

[40] C. Spyrou, G. Samaras, E. Pitoura and P. Evripidou, Mobile agents for wireless computing: the convergence of wireless computational models with mobile-agent technologies, Mobile Networks and Applications 9(5) (October 2004), 517-528.

[41] A.A. Steinmetz and M.G. Kienzle, e-Seminar lecture recording and distribution system. In Multimedia Computing and Networking 4312, SPIE, 2001, pages 25-36.

[42] D. Terry, D. Goldberg, D. Nichols and B. Oki, Continuous queries over append-only databases, ACM SIGMOD Record 21(2) (June 1992), 321-330.

[43] R. Trillo, S. Ilarri and E. Mena, Comparison and performance evaluation of mobile agent platforms. In Third International Conference on Autonomic and Autonomous Systems (ICAS'07), IEEE Computer Society, 2007, pages 41:1-41:6.

[44] A.B. Waluyo, B. Srinivasan and D. Taniar, Global indexing scheme for location-dependent queries in multi channels mobile broadcast environment. In 19th International Conference on Advanced Information Networking and Applications (AINA'05), IEEE Computer Society, 2005, pages 1011-1016.

[45] A.B. Waluyo, B. Srinivasan and D. Taniar, Research on location-dependent queries in mobile databases, International Journal of Computer Systems: Science and Engineering 20(2) (March 2005), 77-93.

[46] J. Wang, Experiential sampling for video surveillance. In First ACM International Workshop on Video Surveillance (IWVS'03), ACM Press, 2003, pages 77-86.

[47] J. Wang, C. Xu, E. Chng, H. Lu and Q. Tian, Automatic composition of broadcast sports video, Multimedia Systems 14(4) (September 2008), 179-193.

[48] O. Wolfson, A.P. Sistla, S. Chamberlain and Y. Yesha, Updating and querying databases that track mobile units, Distributed and Parallel Databases 7(3) (July 1999), 257-287.

[49] X. Xiong, M.F. Mokbel and W.G. Aref, SEA-CNN: Scalable processing of continuous k-nearest neighbor queries in spatio-temporal databases. In 21st International Conference on Data Engineering (ICDE'05), IEEE Computer Society, 2005, pages 643-654.

[50] A. Yilmaz, O. Javed and M. Shah, Object tracking: A survey, ACM Computing Surveys 38(4) (2006), 13:1-13:45.

[51] C. Zhang, Y. Rui, J. Crawford and L.-W. He, An automated end-to-end lecture capture and broadcasting system, ACM Transactions on Multimedia Computing, Communications and, Applications 4(1) (January 2008), 6:1-6:23. 
Sergio Ilarri is an Associate Professor in the Department of Computer Science and Systems Engineering at the University of Zaragoza. He received his B.S. and his Ph.D. in Computer Science from the University of Zaragoza in 2001 and 2006 , respectively. For a year, he was a visiting researcher in the Mobile Computing Laboratory at the Department of Computer Science at the University of Illinois in Chicago, and he has also cooperated (through several research stays) with the University of Valenciennes and with IRIT in Toulouse. His research interests include data management issues for mobile computing, vehicular networks, mobile agents, and the Semantic Web.

Eduardo Mena is an Associate Professor at the University of Zaragoza, Spain. In 1992 he received his B.S. in Computer Science from the University of the Basque Country and in 1999 his Ph.D. degree in Computer Science from the University of Zaragoza. He leads the Distributed Information Systems research group at his university. His research interest areas include interoperable, heterogeneous and distributed information systems, the Semantic Web, and mobile computing.

Arantza Illarramendi is a Full Professor at the Basque Country University, Spain. She received the BSc degree from the Computer Science Faculty of the Technical University of Madrid, Spain, and the Ph.D. degree in Computer Science from the Basque Country University. She leads the BDI research group at the Computer Science Faculty, San Sebastian (Basque Country University). Her main research interests are data management in mobile environments and semantic interoperability among information systems. Her publications include several journal and conference articles. She is a member of the IFIP WG 2.6.

Roberto Yus is a Ph.D. student of Computer Science at the University of Zaragoza, Spain. He received his B.S in Computer Science from the same university in 2011. His research interests concern data management in distributed and mobile environments, semantic data management, and the development of tools for multimedia management and broadcasting.

Maider Laka is a staff researcher member of the GraphicsMediaNet centre Vicomtech-IK4 since 2005. She is a member of the Digital TV \& Multimedia Services Department and her research concerns television production, virtual reality and GIS. Her recent research projects involve enhanced TV production, particularly in sport events.

Gorka Marcos received his diploma degree in Telecommunication Engineering from the University of the Basque Country (UPV-EHU) in December, 2001. In 2002 he started working as full-time scientific staff member for Vicomtech-IK4 technological center. Since then, he has been researching in semantic-based technologies, applying those technologies to improve current approaches for multimedia retrieval. He got a Ph.D. in Computer Science in this field in 2011 and he is an author of multiple international publications. 

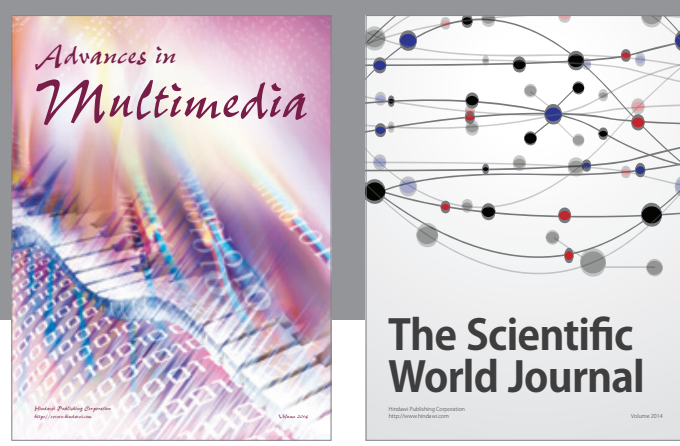

The Scientific World Journal
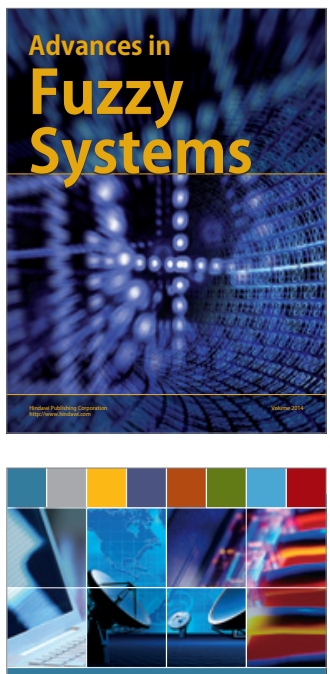

Computer Networks and Communications
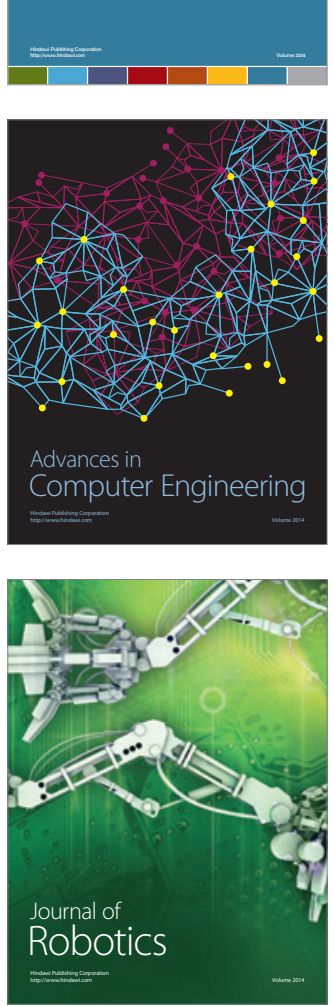
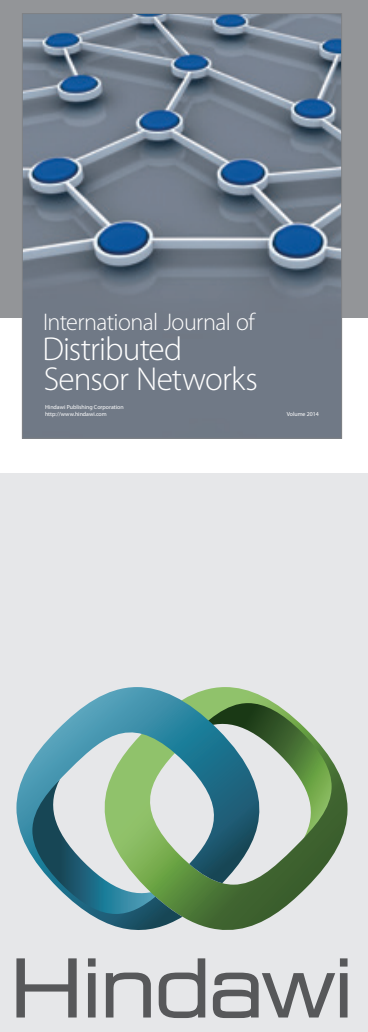

Submit your manuscripts at

http://www.hindawi.com
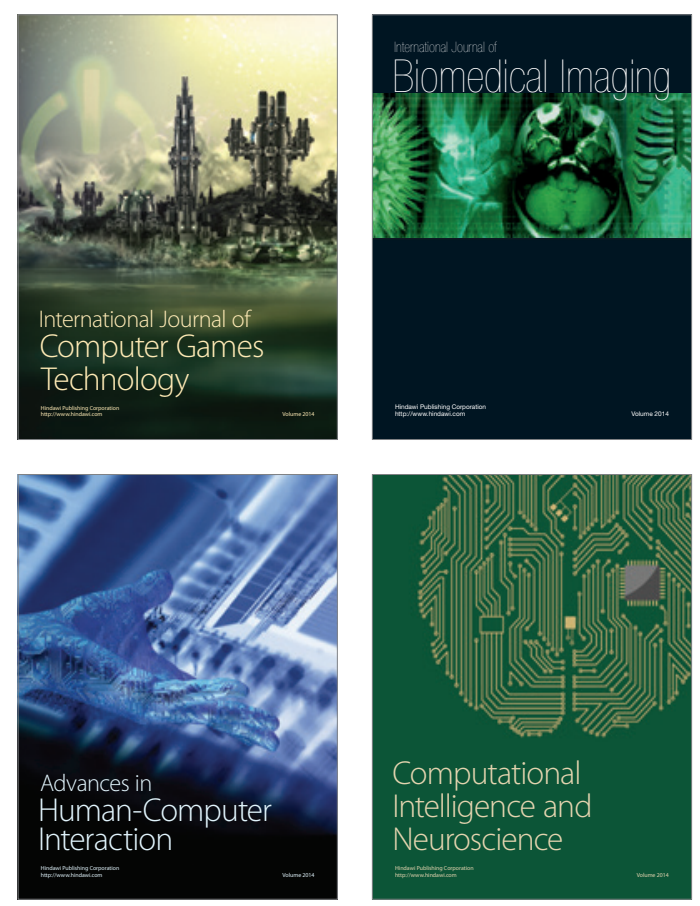
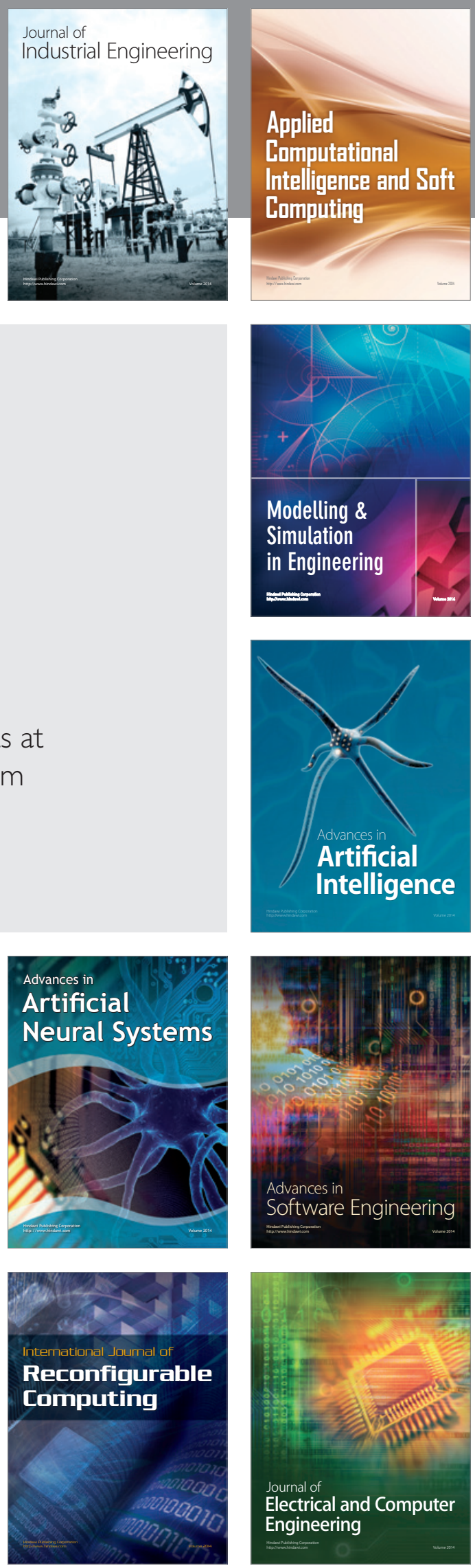